\title{
Feeding ecology, isotopic niche, and ingestion of fishery-related items of the wandering albatross Diomedea exulans at Kerguelen and Crozet Islands
}

\author{
Yves Cherel $^{1, *}$, José C. Xavier ${ }^{2,3}$, Sophie de Grissac ${ }^{1}$, Colette Trouvé ${ }^{1}$, \\ Henri Weimerskirch ${ }^{1}$
}

${ }^{1}$ Centre d'Etudes Biologiques de Chizé, UMR 7372 du CNRS et de l'Université de La Rochelle, 79360 Villiers-en-Bois, France

${ }^{2}$ Marine and Environmental Sciences Centre, Department of Life Sciences, University of Coimbra, 3001-401 Coimbra, Portugal

${ }^{3}$ British Antarctic Survey, Natural Environment Research Council, High Cross, Madingley Road, CB3 0ET Cambridge, UK

\begin{abstract}
Feeding ecology and isotopic niche of the wandering albatross Diomedea exulans were investigated in the poorly studied population on the Kerguelen Islands and compared to that on the Crozet Islands. Fish (48\% by mass) and cephalopods (46\%) were similarly important in chick food at Kerguelen, while cephalopods (87\%) dominated the diet at Crozet. Fish prey included mainly deep-sea species, with the Patagonian toothfish Dissostichus eleginoides being the main item. Cephalopod beaks were identified, most of which were from adult oceanic squids. Albatrosses preyed upon the same taxa at both localities, but in different proportions. Histioteuthis atlantica (30\% by number), Galiteuthis glacialis (13\%), and Kondakovia longimana (10\%) were the main squid prey at Kerguelen, while K. longimana (35\%) and H. eltaninae (23\%) dominated at Crozet. Chick feather $\delta^{15} \mathrm{~N}$ values were higher in wandering albatrosses than in other oceanic seabirds of the 2 communities, indicating that the wandering albatross is an apex consumer together with the sperm whale and sleeper shark that have similar $\delta^{15} \mathrm{~N}$ values. Satellitetracked wandering albatrosses foraged in local subantarctic waters and farther north, with some Crozet birds overlapping with those from the Kerguelen population in western Kerguelen waters. Anthropogenic items (e.g. plastic fragments, hooks) were found in half the food samples. All fishery-related items were from the local toothfish fishery. The high number of hooks from Crozet indicated the presence of a fairly large number of illegal longliners in the area during the Austral winter 1998. A review of the feeding habits of Diomedea spp. highlights the need for more dietary investigations to achieve effective conservation and management of this endangered group of charismatic seabirds.
\end{abstract}

KEY WORDS: Cephalopod $\cdot$ Diet $\cdot$ Hook $\cdot$ Satellite tracking $\cdot$ Seabird $\cdot$ Southern Ocean $\cdot$ Stable isotopes

\section{INTRODUCTION}

Albatrosses of the genus Diomedea are the largest of all living flying birds (wingspan up to $3.5 \mathrm{~m}$ ). The genus comprises 7 species and subspecies including some of the most endangered seabirds, the Amsterdam $D$. amsterdamensis and Tristan $D$. dabbenena albatrosses (Agreement on the Conservation of Albatrosses and Petrels: www.acap.aq/). Diomedea alba-

\footnotetext{
*Corresponding author: cherel@cebc.cnrs.fr
}

trosses breed on scattered islands and archipelagoes of the Southern Ocean and fringing subtropical waters. Only 1 species has a circumpolar distribution: the wandering albatross $D$. exulans, which breeds in the Indian, Pacific, and Atlantic Oceans (Weimerskirch et al. 2015; www.acap.aq/). Its large size and tameness make the wandering albatross an ideal model animal for long-term demographic and biologging investigations. The species is one of the best

(C) The authors 2017. Open Access under Creative Commons by Attribution Licence. Use, distribution and reproduction are unrestricted. Authors and original publication must be credited. 
known seabirds, with most tracking studies having been conducted at 3 locations, namely the Crozet Islands (Jouventin \& Weimerskirch 1990), Marion Island (Nel et al. 2002), and South Georgia (Prince et al. 1992). Its basic biology has been poorly investigated elsewhere, especially at the Kerguelen Islands that host $\sim 15 \%$ of the population worldwide $(\sim 8000$ pairs worldwide; www.acap.aq/).

Wandering albatrosses forage primarily in oceanic waters of the Antarctic, Subantarctic, and Subtropical Zones (Weimerskirch 1998, Xavier et al. 2004), where they feed primarily on fish and on a large diversity of cephalopods (Cherel \& Klages 1998). Albatrosses are strongly attracted to and interact with fishing vessels and, consequently, fishery-related mortality is responsible for alarming declines in their populations (Weimerskirch et al. 1997a, Barbraud et al. 2012). Birds are attracted by offal, bycatch, and the targeted fish, with the primary source of incidental mortality being hooks and the associated baits from longlines (www.acap.aq/). In the 1990s, a demersal longline fishery developed in the southern Atlantic and spread rapidly to other regions of the Southern Ocean (Nel et al. 2002). The fishery targets the Patagonian toothfish Dissostichus eleginoides, a natural prey of wandering albatrosses over their traditional foraging grounds, namely in slope waters surrounding subantarctic islands where the birds nest (Weimerskirch et al. 1997b, Weimerskirch 1998, Lord et al. 2006). Albatrosses congregate in significant numbers in the vicinity of longliners, increasing their risk of being injured or killed during fishing operations (Cherel et al. 1996, Weimerskirch et al. 2000).

The main aim of the present study was to document the diet, foraging ecology, and relationships with fisheries of the wandering albatross population from the Kerguelen Islands. A combination of methods (satellite tracking, stomach content analysis, and stable isotopes) was used to make direct comparisons with the better known Crozet Island population, which was sampled in the same years using the same methods as the Kerguelen population. Foraging areas of breeding birds were investigated by performing the first satellite-tracking of wandering albatrosses at the Kerguelen Islands. Stomach contents were collected in the same year (1998) that corresponded to the peak in the Patagonian toothfish fishery in Kerguelen waters, with illegal longlining representing $\sim 70 \%$ of the catch (Lord et al. 2006). Consequently, relationships with fisheries were investigated through careful examination of anthropogenic items in food samples. Stomach content analy- sis of large chicks allowed identification and quantification of prey species and prey size, and potential seasonal dietary changes were investigated during the long chick-rearing period of the species (from March to December; www.acap.aq/). The feeding habits of albatrosses in the genus Diomedea were then reviewed from the literature to highlight the key prey groups and prey species, but also the lack of dietary information on this endangered seabird taxon. Finally, stable isotopes were used as a complementary indirect method to delineate the foraging habitats $\left(\delta^{13} \mathrm{C}\right.$ value as a proxy) and trophic levels $\left(\delta^{15} \mathrm{~N}\right.$ value as a proxy) of the wandering albatross. We focused on the trophic position of the species relative to other Procellariiformes and to other consumers to study the trophic structure of the seabird community and of the whole ecosystem, respectively. Recent investigations showed that wandering albatrosses are apex consumers of the subantarctic seabird assemblages (Jaeger et al. 2013), but, to our knowledge, no information is available at the ecosystem level. Wandering albatrosses forage on the same prey as some marine mammals and predatory fish, suggesting a closely related trophic level with the larger sleeper sharks and sperm whales (Cherel \& Duhamel 2004).

\section{MATERIALS AND METHODS}

\section{Study site, birds, and dietary sampling and analysis}

Dietary studies were conducted in the same year (1998) at the Kerguelen $\left(49^{\circ} \mathrm{S}, 70^{\circ} \mathrm{E}\right)$ and Crozet $\left(46^{\circ} \mathrm{S}, 51^{\circ} \mathrm{E}\right.$; Possession Island) archipelagoes in the southern Indian Ocean. Stomach contents from large chicks were collected at Kerguelen $(\mathrm{n}=30)$ and Crozet Islands $(n=33)$. Due to the long chick-rearing period of the wandering albatross, $\sim 10$ large chicks were sampled when they were $\sim 3,5$, and 7 mo old (June, August, and October) to assess time-related dietary changes. Food samples were taken from randomly selected chicks either after a returning parent had completed feeding them, or after successive daily weighing indicated that they had been fed recently. Samples were obtained by up-ending chicks over a plastic bucket and massaging the stomach and throat. If needed, chicks were flushed once using the offloading technique (Wilson 1984). Collection of a single meal has no detrimental effects in terms of survival and development of large albatross chicks (Phillips 2006). Food samples were all returned deep-frozen $\left(-20^{\circ} \mathrm{C}\right)$ to the laboratory in France for analysis. 
Each sample was thawed, drained, and weighed (solid fraction; Cherel et al. 2000). Accumulated items, mostly cephalopod beaks and spermatophores, were subsequently sorted and weighed. Beaks can persist in predator stomachs for weeks and even months, thus overemphasizing their importance in seabird diets (Xavier et al. 2005). Following Cherel \& Klages (1998), accumulated beaks (without flesh attached) were consequently analyzed separately from fresh items. Fresh items (solid fraction minus accumulated items) were divided into broad prey classes (fish, cephalopods, crustaceans, carrion, and others), which were weighed to calculate their proportion by mass in the diet. Species identification of prey relied almost entirely on the examination of otoliths and bones for fish, chitinized beaks for cephalopods, and exoskeletons for crustaceans. Special care was taken to use all fish hard parts recovered in stomach contents (bones, cartilaginous elements, otoliths, scales, thorns, teeth), with an emphasis on some distinctive bones (premaxilla, maxilla, dentary, articular, parasphenoid, opercle, vertebrae, and caudal skeleton) to identify items to the lowest possible taxon. In the same way, the morphology of both lower and upper beaks, instead of that of lower beaks only, was used to determine cephalopod prey (Cherel et al. 2000, Xavier et al. 2011). It was not possible to differentiate upper beaks of the common Histioteuthis atlantica from those of the rarer $H$. bonnellii corpuscula.

Items were identified by comparison with material held in our own collection and by reference to the available literature, including Andersen (1984), Williams \& McEldowney (1990), and Duhamel et al. (2005) for fish, Murano (1999) for crustaceans, and Clarke (1986) and Xavier \& Cherel (2009) for cephalopods. Species names of cephalopods followed either recent and ongoing taxonomic revisions (Young et al. 2016) or well-known beaks with still no species correspondence (Clarke 1986, Imber 1992, Cherel et al. 2004). Length of uneroded or slightly eroded otoliths (OL) and of dentary bones (ML for mandible length) of fish, lower rostral length (LRL) of squid beaks, and lower hood length (LHL) of octopus beaks were measured with a Vernier caliper. Fish standard or total length (SL or TL), cephalopod dorsal mantle length (DML), and prey mass were calculated using regression equations (Clarke 1986, Williams \& McEldowney 1990, Smale et al. 1995, Lu \& Ickeringill 2002, Xavier \& Cherel 2009). For the few species where no relationships were available, lengths were estimated using equations for closely related species or for species with a similar morphology.

\section{Satellite tracking}

Nine and 45 breeding albatrosses were satellitetracked in 1998 during the brooding period (April) and during the incubation and brooding periods (January to April) at Kerguelen and Crozet Islands, respectively. The remoteness of albatross nests at Kerguelen made the field work more difficult compared to the more accessible, and thus more investigated, birds from Crozet (Weimerskirch et al. 1993). The difficulty of recovering satellite tags during the chick-rearing period, when birds stay briefly near their nest, precluded working in June to October. Hence, there was a temporal mismatch between the tracking period (late incubation and brooding) and the period of dietary sampling (mid- to late-chick rearing). The 2 periods are marked by different feeding behaviors, with wandering albatrosses increasing their foraging range in winter (Weimerskirch et al. 1993). Wandering albatrosses were fitted with satellite transmitters (Microwave 100 and T2038 Toyocom) weighing between 55 and $80 \mathrm{~g}$, i.e. below the $3 \%$ body mass limit recommended for flying birds (Phillips et al. 2003). The transmitters were fitted on back feathers with adhesive tape and glue. They were left on the birds for 1 to 3 successive foraging trips. Satellite fixes were obtained through the Argos system. All locations (classes A, B, 0, and 1 to 3) were used, but unrealistic positions were filtered out by removing those with an estimated speed above $90 \mathrm{~km} \mathrm{~h}^{-1}$ (McConnell et al. 1992; details in Weimerskirch et al. 1993).

Kernel analysis was used to map the density distribution of the 2 populations. Serial independence of the locations is not required with this method (de Solla et al. 1999), but homogeneous sampling frequency is needed (Wood et al. 2000). Thus, satellite fixes on a regular 2 daily fixes basis were re-sampled to deal with irregular Argos transmissions. Kernels were calculated using the kernelUD function in the 'adehabitatHR' R package (Calenge 2006), with a smoothing parameter value of 0.5 to account for satellite transmitter error. Ninety-five percent density contours are considered to represent the home range (Gallerani Lawson \& Rodgers 1997, Brothers et al. 1998), and 75 and $50 \%$ density contours to indicate outer and inner core areas, respectively. Maps were generated using the 'maps' $\mathrm{R}$ package. Overlaid bathymetry was downloaded from the NOAA Coast Watch website (http://coastwatch.pfeg.noaa. gov/erddap/index.html). Position of the Polar (PF) and Subtropical Fronts (STF) was estimated from Aviso products (Altimetric Sea Level Anomaly; 
http://ctoh.legos.obs-mip.fr/applications/mesoscale/ southern-ocean-fronts; Sallee et al. 2008) and from data in Belkin \& Gordon (1996), respectively.

\section{Stable isotope analysis}

Chick $\delta^{13} \mathrm{C}$ and $\delta^{15} \mathrm{~N}$ values are good proxies to compare habitats and trophic positions within seabird communities, because they integrate the isotopic values of assimilated food caught in the vicinity of the colonies by parent birds during the breeding season. Body feathers were collected from large Procellariiformes, including albatrosses and giant petrels, which breed at Kerguelen and Crozet Islands. For each study species, 4 dorsal body feathers were sampled from randomly chosen chicks at the end of the chick-rearing period. To avoid potential interannual variation in $\delta^{13} \mathrm{C}$ and $\delta^{15} \mathrm{~N}$ values linked to a shift in baseline values (Jaeger \& Cherel 2011), all feathers were sampled in the same year (2005), which was different from the year of tracking and stomach sampling (1998). In Procellariiformes, body feathers of chicks grow almost synchronously in the mid- to the second half of the chick-rearing period and thus present low intra-individual isotopic variation (Phillips \& Hamer 2000, Carravieri et al. 2014). Hence, $\delta^{13} \mathrm{C}$ and $\delta^{15} \mathrm{~N}$ values were measured for a single feather per individual. Prior to isotopic analysis, feathers were cleaned of surface lipids and contaminants by immersion in a solution of $2: 1$ chloroform:methanol for $2 \mathrm{~min}$ in a beaker, followed by 2 successive methanol rinses. Each whole body feather was air dried, calamus was removed, and the feather was cut into small pieces with scissors. Tissue $\delta^{15} \mathrm{~N}$ values were also compared between the wandering albatross and other non-avian predators from the Kerguelen Islands, namely female elephant seals Mirounga leonina ( $\mathrm{n}=32$, whole blood, Cherel et al. 2008), female Antarctic fur seals Arctocephalus gazella ( $\mathrm{n}=10$, whole blood, Cherel et al. 2008), Patagonian toothfish $(\mathrm{n}=36$, muscle), porbeagle sharks Lamna nasus ( $\mathrm{n}=7$, muscle), sleeper sharks Somniosus antarcticus ( $\mathrm{n}=20$, muscle), and sperm whales Physeter macrocephalus ( $\mathrm{n}=3$, skin). Muscle and skin samples were freeze-dried and ground in a mortar, and lipids were subsequently extracted using cyclohexane. Tissue sub-samples were then weighed ( $0.4 \mathrm{mg}$ ) with a microbalance, packed in tin containers, and nitrogen and carbon isotope ratios were determined by a continuous flow mass spectrometer (Micromass Isoprime) coupled to an elemental analyzer (Euro Vector EA 3024). Results are presented in the usual $\delta$ notation relative to Vienna PeeDee Belemnite and atmospheric $\mathrm{N}_{2}$ for $\delta^{13} \mathrm{C}$ and $\delta^{15} \mathrm{~N}$, respectively. Replicate measurements of internal laboratory standards (acetanilide) indicated measurement errors $<0.15$ and $<0.20 \%$ for $\delta^{13} \mathrm{C}$ and $\delta^{15} \mathrm{~N}$, respectively.

The isotopic method was validated in the southern Indian Ocean. $\delta^{13} \mathrm{C}$ values of consumers reflect the latitudinal $\delta^{13} \mathrm{C}$ gradient at the base of the food web and thus indicate their latitudinal foraging habitats (Cherel \& Hobson 2007, Jaeger et al. 2010), while $\delta^{15} \mathrm{~N}$ values change according to their trophic position in the increasing order planktivorous species < piscivorous species < fish and squid eaters (Cherel et al. 2010). To help interpret $\delta^{15} \mathrm{~N}$ values of Procellariiformes, they were compared to values of body feathers from a consumer known to feed on mesopelagic fish, namely chicks of the myctophid-eating king penguin Aptenodytes patagonicus (Cherel et al. 2010). Since isotopic discrimination factors are tissue specific, isotopic comparisons amongst tissues require correcting raw isotopic values. $\delta^{15} \mathrm{~N}$ values from Kerguelen consumers were measured on various tissues, including whole blood, muscle, skin, and feathers, which, indeed, present different $\delta^{15} \mathrm{~N}$ discrimination factors (Vanderklift \& Ponsard 2003). However, an analysis using corrected (estimated) $\delta^{15} \mathrm{~N}$ values did not fundamentally alter the main isotopic finding of the study (the 2 clusters of species, see below); hence, statistical analysis was performed on the raw (measured) $\delta^{15} \mathrm{~N}$ values. All data were statistically analyzed using SYSTAT 13. Values are presented as means $\pm \mathrm{SD}$.

\section{RESULTS \\ Diet \\ Kerguelen}

The fresh component of the stomach contents of large wandering albatross chicks was composed almost equally of fish (48\% by mass, $35 \%$ by number, all 30 samples pooled) and cephalopods (46 and $30 \%$, respectively; Tables $1 \& 2$ ). Other food sources were negligible by mass, but crustaceans and gelatinous plankton accounted for a significant number of prey (13 and $15 \%$, respectively).

We identified 112 fresh prey items (Table 2). The main fish was the Patagonian toothfish Dissostichus eleginoides (19\% by number), with other species including skates and the morid Antimora rostrata. All 
Table 1. Mass and composition of stomach contents of wandering albatross Diomedea exulans chicks at Kerguelen and Crozet Islands in 1998. Values are means \pm SD. A few stomach samples contained no or very few accumulated items, thus explaining the lower number of samples that were used to calculate the mean number of accumulated beaks per sample. Mann-Whitney $U$-tests were performed to compare the 2 localities, and Kruskal-Wallis $H$-tests compared seasons within each locality. Significant differences $(\mathrm{p}<0.05)$ are highlighted in bold; na: not applicable

\begin{tabular}{|c|c|c|c|c|c|c|c|c|}
\hline & \multirow[t]{2}{*}{ Total } & \multicolumn{2}{|c|}{$\begin{array}{l}\text { Statistics } \\
\text { Kerguelen } \\
\text { vs. Crozet }\end{array}$} & \multirow[t]{2}{*}{$\begin{array}{l}\text { June } \\
1998\end{array}$} & \multirow[t]{2}{*}{$\begin{array}{c}\text { August } \\
1998\end{array}$} & \multirow[t]{2}{*}{$\begin{array}{c}\text { October } \\
1998\end{array}$} & \multicolumn{2}{|c|}{$\begin{array}{c}\text { Statistics } \\
\text { Temporal } \\
\text { changes }\end{array}$} \\
\hline & & $U$ & $\mathrm{p}$ & & & & $H$ & $\mathrm{p}$ \\
\hline \multicolumn{9}{|l|}{ Kerguelen } \\
\hline Stomach samples (n) & 30 & & & 10 & 10 & 10 & & \\
\hline Solid fraction $(g)$ & $388 \pm 185$ & & & $353 \pm 182$ & $461 \pm 205$ & $350 \pm 162$ & 1.9 & 0.391 \\
\hline Accumulated items (g) & $54 \pm 29$ & & & $35 \pm 25$ & $51 \pm 16$ & $76 \pm 30$ & 9.2 & 0.010 \\
\hline Fresh items $(g)$ & $334 \pm 181$ & & & $318 \pm 175$ & $409 \pm 200$ & $275 \pm 157$ & 2.2 & 0.326 \\
\hline Fish $(g)$ & $161 \pm 203$ & & & $175 \pm 217$ & $181 \pm 242$ & $128 \pm 159$ & 0.3 & 0.865 \\
\hline Cephalopods (g) & $152 \pm 158$ & & & $126 \pm 144$ & $192 \pm 171$ & $140 \pm 165$ & 0.8 & 0.676 \\
\hline Crustaceans (g) & $0.4 \pm 1.0$ & & & $0.7 \pm 1.6$ & $0.2 \pm 0.4$ & $0.3 \pm 0.6$ & 0.3 & 0.853 \\
\hline Carrion $(\mathrm{g})$ & $9.2 \pm 27.8$ & & & $16.5 \pm 40.9$ & $9.7 \pm 26.3$ & $1.4 \pm 3.0$ & 0.1 & 0.966 \\
\hline Others $(\mathrm{g})$ & $10.7 \pm 33.4$ & & & $0.2 \pm 0.7$ & $26.9 \pm 53.7$ & $5.0 \pm 15.9$ & 5.9 & 0.051 \\
\hline \multicolumn{9}{|l|}{ Overall composition: } \\
\hline Fish (\%) & 48.3 & & & 55.0 & 44.1 & 46.7 & na & na \\
\hline Cephalopods (\%) & 45.6 & & & 39.5 & 46.9 & 50.8 & na & na \\
\hline Crustaceans (\%) & 0.1 & & & 0.2 & $<0.1$ & 0.1 & na & na \\
\hline Carrion (\%) & 2.8 & & & 5.2 & 2.4 & 0.5 & na & na \\
\hline Others $(\%)$ & 3.2 & & & $<0.1$ & 6.6 & 1.8 & na & na \\
\hline Accumulated beaks (g) & $26.9 \pm 22.0$ & & & $8.3 \pm 6.6$ & $24.0 \pm 14.9$ & $48.4 \pm 19.6$ & 15.9 & $<0.0001$ \\
\hline Accumulated beaks (n) & (27) $192 \pm 108$ & & & (8) $101 \pm 35$ & (10) $158 \pm 41$ & (9) $311 \pm 96$ & 19.5 & $<0.0001$ \\
\hline \multicolumn{9}{|l|}{ Crozet } \\
\hline Stomach samples (n) & 33 & & & 10 & 12 & 11 & & \\
\hline Solid fraction $(\mathrm{g})$ & $673 \pm 322$ & 758 & $<0.0001$ & $836 \pm 297$ & $766 \pm 281$ & $424 \pm 245$ & 9.5 & 0.009 \\
\hline Accumulated items (g) & $104 \pm 59$ & 771 & $<0.0001$ & $62 \pm 27$ & $115 \pm 49$ & $130 \pm 72$ & 9.1 & 0.011 \\
\hline Fresh items $(g)$ & $569 \pm 325$ & 720 & 0.002 & $774 \pm 285$ & $651 \pm 275$ & $294 \pm 222$ & 12.0 & 0.003 \\
\hline Fish $(g)$ & $68 \pm 184$ & 309 & 0.009 & $84 \pm 185$ & $105 \pm 254$ & $13 \pm 26$ & 3.5 & 0.174 \\
\hline Cephalopods (g) & $497 \pm 286$ & 851 & $<0.0001$ & $689 \pm 265$ & $536 \pm 228$ & $280 \pm 226$ & 10.2 & 0.006 \\
\hline Crustaceans (g) & $0.4 \pm 1.4$ & 428 & 0.202 & $0.7 \pm 2.0$ & $0.3 \pm 0.7$ & $0.5 \pm 1.5$ & 0.4 & 0.805 \\
\hline Carrion (g) & $3.4 \pm 16.0$ & 428 & 0.111 & 0.0 & $9.4 \pm 26.2$ & 0.0 & na & na \\
\hline Others (g) & $0.2 \pm 0.7$ & 404 & 0.039 & 0.0 & 0.0 & $0.5 \pm 1.2$ & na & na \\
\hline \multicolumn{9}{|l|}{ Overall composition: } \\
\hline Fish $(\%)$ & 11.9 & na & na & 10.9 & 16.1 & 4.3 & na & na \\
\hline Cephalopods (\%) & 87.4 & na & na & 89.0 & 82.4 & 95.4 & na & na \\
\hline Crustaceans (\%) & $<0.1$ & na & na & $<0.1$ & $<0.1$ & 0.2 & na & na \\
\hline Carrion $(\%)$ & 0.6 & na & na & 0.0 & 1.4 & 0.0 & na & na \\
\hline Others $(\%)$ & $<0.1$ & na & na & 0.0 & 0.0 & 0.2 & na & na \\
\hline Accumulated beaks (g) & $48.1 \pm 37.6$ & 677 & 0.012 & $18.1 \pm 7.2$ & $51.3 \pm 35.0$ & $71.9 \pm 39.8$ & 13.6 & 0.001 \\
\hline Accumulated beaks (n) & (30) $248 \pm 133$ & 518 & 0.071 & (10) $136 \pm 56$ & (10) $266 \pm 76$ & (10) $343 \pm 154$ & 14.7 & 0.001 \\
\hline
\end{tabular}

cephalopods were oceanic squids, with the main item being the onychoteuthid Kondakovia longimana $(10 \%)$. Other squids included the histioteuthid Histioteuthis atlantica and the cranchiid Taonius sp. B (Voss). A significant number of the pelagic tunicate Pyrosoma atlantica was found in 3 food samples. Crustacean prey included large deep-sea mysids and females of large copepods that parasitize fish. Seasonal changes were observed neither in the mass of the solid and fresh fractions of food samples nor in the mass of the main prey groups, but sample sizes were relatively low (Table 1).

\section{Crozet}

The food of albatrosses from Crozet Islands was overall dominated by cephalopods $(87 \%$ by fresh mass, $58 \%$ by number), with fish ranking second (12 and $29 \%$, respectively; Tables 1 \& 2). Fresh remains of cephalopods were found in all 33 samples. Other prey groups were negligible. We identified 119 fresh prey items (Table 2). All cephalopods were oceanic squids, with the main item being $K$. longimana (35\% by number). Other squids included the ommastrephid Martialia hyadesi, the onychoteuthid Onykia in- 
Table 2. Frequency of occurrence and numbers of fresh prey items identified from stomach contents of wandering albatross Diomedea exulans chicks at Kerguelen and Crozet Islands in 1998

\begin{tabular}{|c|c|c|c|c|c|c|c|c|}
\hline \multirow[t]{3}{*}{ Species } & \multicolumn{4}{|c|}{ Kerguelen (n=28) } & \multicolumn{4}{|c|}{ Crozet $(\mathrm{n}=33$ ) } \\
\hline & \multicolumn{2}{|c|}{ Occurrence } & \multicolumn{2}{|c|}{ Number } & \multicolumn{2}{|c|}{ Occurrence } & \multicolumn{2}{|c|}{ Number } \\
\hline & $\mathrm{n}$ & $\%$ & $\mathrm{n}$ & $\%$ & $\mathrm{n}$ & $\%$ & $\mathrm{n}$ & $\%$ \\
\hline Carrion & 7 & 25.0 & 7 & 6.3 & 2 & 6.1 & 2 & 1.7 \\
\hline Unidentified carrion & 7 & 25.0 & 7 & 6.3 & 2 & 6.1 & 2 & 1.7 \\
\hline Fish & 25 & 89.3 & 39 & 34.8 & 19 & 57.6 & 34 & 28.6 \\
\hline \multicolumn{9}{|l|}{ Rajidae } \\
\hline Bathyraja sp. & 4 & 14.3 & 4 & 3.6 & & & & \\
\hline \multicolumn{9}{|l|}{ Paralepididae } \\
\hline Magnisudis prionosa & 1 & 3.6 & 1 & 0.9 & 1 & 3.0 & 1 & 0.8 \\
\hline \multicolumn{9}{|l|}{ Myctophidae } \\
\hline Lampadena notialis & 1 & 3.6 & 1 & 0.9 & & & & \\
\hline \multicolumn{9}{|l|}{ Muraenolepididae } \\
\hline Muraenolepis marmoratus & 1 & 3.6 & 1 & 0.9 & 2 & 6.1 & 2 & 1.7 \\
\hline \multicolumn{9}{|l|}{ Macrouridae } \\
\hline Macrourus carinatus/holotrachys & 1 & 3.6 & 1 & 0.9 & 2 & 6.1 & 3 & 2.5 \\
\hline \multicolumn{9}{|l|}{ Moridae } \\
\hline Antimora rostrata & 2 & 7.1 & 2 & 1.8 & 2 & 6.1 & 2 & 1.7 \\
\hline Halargyreus johnsonii & & & & & 1 & 3.0 & 1 & 0.8 \\
\hline \multicolumn{9}{|l|}{ Oreosomatidae } \\
\hline Unidentified Oreosomatidae & & & & & 2 & 6.1 & 2 & 1.7 \\
\hline \multicolumn{9}{|l|}{ Nototheniidae } \\
\hline Dissostichus eleginoides & 19 & 67.9 & 21 & 18.8 & 16 & 48.5 & 18 & 15.1 \\
\hline Channichthyidae & & & & & & & & \\
\hline Champsocephalus gunnari & 1 & 3.6 & 1 & 0.9 & & & & \\
\hline Unidentified fish & 5 & 17.9 & 7 & 6.3 & 5 & 15.2 & 5 & 4.2 \\
\hline Cephalopods & 23 & 82.1 & 34 & 30.4 & 33 & 100.0 & 69 & $\mathbf{5 8 . 0}$ \\
\hline Ommastrephidae & & & & & & & & \\
\hline Martialia hyadesi & 2 & 7.1 & 2 & 1.8 & 6 & 18.2 & 6 & 5.0 \\
\hline Onychoteuthidae & & & & & & & & \\
\hline Kondakovia longimana & 6 & 21.4 & 11 & 9.8 & 20 & 60.6 & 42 & 35.3 \\
\hline Onykia ingens & & & & & 3 & 9.1 & 3 & 2.5 \\
\hline Gonatidae & & & & & & & & \\
\hline Gonatus antarcticus & & & & & 1 & 3.0 & 1 & 0.8 \\
\hline Histioteuthidae & & & & & & & & \\
\hline Histioteuthis atlantica & 3 & 10.7 & 4 & 3.6 & & & & \\
\hline Histioteuthis eltaninae & & & & & 2 & 6.1 & 2 & 1.7 \\
\hline Histioteuthis miranda & & & & & 1 & 3.0 & 1 & 0.8 \\
\hline Batoteuthidae & & & & & & & & \\
\hline Batoteuthis skolops & & & & & 2 & 6.1 & 3 & 2.5 \\
\hline Cranchiidae & & & & & & & & \\
\hline Taonius sp. B (Voss) & 2 & 7.1 & 5 & 4.5 & 1 & 3.0 & 1 & 0.8 \\
\hline Unidentified squid & 12 & 42.9 & 12 & 10.7 & 10 & 30.3 & 10 & 8.4 \\
\hline Crustaceans & 9 & 32.1 & 15 & 13.4 & 5 & 15.2 & 7 & 5.9 \\
\hline Mysida & & & & & & & & \\
\hline Neognathophausia ingens & 3 & 10.7 & 3 & 2.7 & 2 & 6.1 & 2 & 1.7 \\
\hline Neognathophausia gigas & & & & & 1 & 3.0 & 1 & 0.8 \\
\hline Unidentified Neognathophausia & 5 & 17.9 & 5 & 4.5 & & & & \\
\hline Amphipoda & & & & & & & & \\
\hline Themisto gaudichaudii & & & & & 1 & 3.0 & 1 & 0.8 \\
\hline Copepoda & & & & & & & & \\
\hline Unidentified Lophoura & 1 & 3.6 & 1 & 0.9 & 2 & 6.1 & 2 & 1.7 \\
\hline Unidentified Sphyrion & 2 & 7.1 & 6 & 5.4 & & & & \\
\hline Unidentified crustacean & & & & & 1 & 3.0 & 1 & 0.8 \\
\hline Others & 4 & 14.3 & 17 & 15.2 & 2 & 6.1 & 7 & 5.9 \\
\hline Pyrosomida & & & & & & & & \\
\hline Pyrosoma atlantica & 3 & 10.7 & 16 & 14.3 & 2 & 6.1 & 7 & 5.9 \\
\hline Unidentified gelatinous plankton & 1 & 3.6 & 1 & 0.9 & & & & \\
\hline Total & & & 112 & 100.0 & & & 119 & 100.0 \\
\hline
\end{tabular}

gens, and the batoteuthid Batoteuthis skolops. The only significant fish prey was the Patagonian toothfish $(15 \%)$, with other species including the rattail Macrourus carinatus/holotrachys, morids, and oreosomatids. The tunicate $P$. atlantica was found in 2 food samples. Crustacean prey included large mysids and parasitic copepods.

Seasonal mass variations were observed in various components of the food samples. While mass of accumulated items increased with time, masses of the solid fraction and fresh items decreased from June to October (by 49 and $62 \%$, respectively). The decreases resulted from a $59 \%$ drop in the mass of cephalopods in food samples over the chick-rearing period, with no concomitant mass changes in other prey groups (Table 1).

\section{Comparison between sites}

The mass of food samples (solid fraction) was overall $73 \%$ higher at Crozet than at Kerguelen (Table 1). The difference was driven by cephalopods rather than fish, with mass of fresh squids being 3.3 times higher in Crozet than Kerguelen food samples. Accordingly, masses of accumulated items and of accumulated beaks were higher in Crozet samples, as was the number of accumulated cephalopod beaks.

Plastic particles were found in 15 $(50 \%)$ and fishery-related items (bait remains and hooks/snoods) were found in $18(55 \%)$ stomach contents from Kerguelen and Crozet Islands. Only 1 Kerguelen sample contained 1 hook, thus contrasting with the higher hook prevalence in Crozet samples (17 hooks in 13 [39\%] samples, up to 4 hooks in 1 sample, equality of proportion tests, $Z=3.44, \mathrm{p}=0.001$ ). Notably, some hooks were intact, but most were partially corroded. Hooks were attached or not with their corresponding snoods (branch lines). In a few cases, the past presence of hooks 
in food samples was indicated by the finding of knotted snoods without attached hooks, meaning that the hooks had been completely digested in the birds' stomach after ingestion.

\section{Accumulated cephalopod beaks}

The mass and number of accumulated beaks increased from June to October at both localities (Table 1). A total of 12860 accumulated beaks were sorted and analyzed from the 63 food samples. The number included 5118 and 7422 identifiable beaks from Kerguelen and Crozet Islands, respectively. Fifty different cephalopod taxa were identified, with many more squids (Oegopsida, 47 taxa) than octopu- ses (Octopoda, 3 taxa; Table 3). Almost all beaks were fully darkened, indicating that they belonged to adult individuals and not to juvenile and immature specimens.

Table 3. Cephalopod diet of the wandering albatross Diomedea exulans at Kerguelen and Crozet Islands in 1998. Values are frequency of occurrence and numbers of accumulated upper and lower beaks that were sorted from stomach samples of large chicks. Equality of proportion tests (large-sample tests) were performed to compare relative beak numbers between the 2 localities. Significant differences $(\mathrm{p}<0.05)$ are highlighted in bold; na: not applicable

\begin{tabular}{|c|c|c|c|c|c|c|c|c|c|c|}
\hline \multirow[t]{3}{*}{ Species } & \multicolumn{4}{|c|}{ Kerguelen $(\mathrm{n}=30)$} & \multicolumn{4}{|c|}{ Crozet $(n=33)$} & \multirow{2}{*}{\multicolumn{2}{|c|}{$\begin{array}{l}\text { Statistics on } \\
\text { numbers }(\%)\end{array}$}} \\
\hline & \multicolumn{2}{|c|}{ Occurrence } & \multicolumn{2}{|c|}{ Number } & \multicolumn{2}{|c|}{ Occurrence } & \multicolumn{2}{|c|}{ Number } & & \\
\hline & $\mathrm{n}$ & $\%$ & $\mathrm{n}$ & $\%$ & $\mathrm{n}$ & $\%$ & $\mathrm{n}$ & $\%$ & $Z$ & $\mathrm{p}$ \\
\hline \multicolumn{11}{|l|}{ Decapoda } \\
\hline \multicolumn{11}{|l|}{ Architeuthidae } \\
\hline Architeuthis dux & 21 & 70.0 & 50 & 1.0 & 5 & 15.2 & 9 & 0.1 & 6.88 & $<0.0001$ \\
\hline \multicolumn{11}{|l|}{ Ommastrephidae } \\
\hline Illex argentinus (bait) & & & & & 3 & 9.1 & 6 & 0.1 & na & na \\
\hline Martialia hyadesi & 29 & 96.7 & 246 & 4.8 & 32 & 97.0 & 369 & 5.0 & 0.42 & 0.674 \\
\hline Todarodes sp. & 16 & 53.3 & 65 & 1.3 & 3 & 9.1 & 9 & 0.1 & 8.26 & $<0.0001$ \\
\hline \multicolumn{11}{|l|}{ Onychoteuthidae } \\
\hline Filippovia knipovitchi & 26 & 86.7 & 132 & 2.6 & 31 & 93.9 & 261 & 3.5 & 2.96 & 0.003 \\
\hline Kondakovia longimana & 28 & 93.3 & 531 & 10.4 & 33 & 100.0 & 2594 & 35.0 & 31.27 & $7<0.0001$ \\
\hline Moroteuthopsis sp. B (Imber) & 6 & 20.0 & 10 & 0.2 & & & & & na & na \\
\hline Notonykia africanae & 1 & 3.3 & 2 & $<0.1$ & 3 & 9.1 & 5 & 0.1 & 0.66 & 0.510 \\
\hline Onychoteuthis banksii complex & 2 & 6.7 & 5 & 0.1 & 1 & 3.0 & 1 & $<0.1$ & 2.12 & 0.034 \\
\hline Onychoteuthis sp. 2 (Imber) & 1 & 3.3 & 1 & $<0.1$ & 1 & 3.0 & 1 & $<0.1$ & 0.26 & 0.792 \\
\hline Onychoteuthis sp. C (Imber) & 12 & 40.0 & 20 & 0.4 & 2 & 6.1 & 3 & $<0.1$ & 4.51 & $<0.0001$ \\
\hline Onykia ingens & 8 & 26.7 & 16 & 0.3 & 33 & 100.0 & 332 & 4.5 & 13.94 & $4<0.0001$ \\
\hline Onykia robsoni & 16 & 53.3 & 47 & 0.9 & 3 & 9.1 & 5 & 0.1 & 7.29 & $<0.0001$ \\
\hline \multicolumn{11}{|l|}{ Pholidoteuthidae } \\
\hline Pholidoteuthis massyae & & & & & 1 & 3.0 & 2 & $<0.1$ & na & na \\
\hline \multicolumn{11}{|l|}{ Psychroteuthidae } \\
\hline Psychroteuthis glacialis & 3 & 10.0 & 7 & 0.1 & & & & & na & na \\
\hline \multicolumn{11}{|l|}{ Brachioteuthidae } \\
\hline Slosarczykovia circumantarctica & & & & & 4 & 12.1 & 4 & 0.1 & na & na \\
\hline \multicolumn{11}{|l|}{ Gonatidae } \\
\hline Gonatus antarcticus & 22 & 73.3 & 84 & 1.6 & 22 & 66.7 & 108 & 1.5 & 0.83 & 0.404 \\
\hline \multicolumn{11}{|l|}{ Ancistrocheiridae } \\
\hline Ancistrocheirus lesueurii & 13 & 43.3 & 34 & 0.7 & 8 & 24.2 & 14 & 0.2 & 4.24 & $<0.0001$ \\
\hline \multicolumn{11}{|l|}{ Lycoteuthidae } \\
\hline Lycoteuthis lorigera & & & & & 2 & 6.1 & 2 & $<0.1$ & na & na \\
\hline \multicolumn{11}{|l|}{ Octopoteuthidae } \\
\hline Octopoteuthis sp. & 9 & 30.0 & 47 & 0.9 & 2 & 6.1 & 3 & $<0.1$ & 7.67 & $<0.0001$ \\
\hline Taningia danae & 16 & 53.3 & 80 & 1.6 & 6 & 18.2 & 8 & 0.1 & 9.60 & $<0.0001$ \\
\hline \multicolumn{11}{|l|}{ Lepidoteuthidae } \\
\hline Lepidoteuthis grimaldii & 2 & 6.7 & 3 & 0.1 & 6 & 18.2 & 13 & 0.2 & 1.80 & 0.072 \\
\hline
\end{tabular}


Table 3 (continued)

\begin{tabular}{|c|c|c|c|c|c|c|c|c|c|c|}
\hline \multirow[t]{3}{*}{ Species } & \multicolumn{4}{|c|}{ Kerguelen $(\mathrm{n}=30)$} & \multicolumn{4}{|c|}{ Crozet $(\mathrm{n}=33)$} & \multirow{2}{*}{\multicolumn{2}{|c|}{$\begin{array}{l}\text { Statistics on } \\
\text { numbers }(\%)\end{array}$}} \\
\hline & \multicolumn{2}{|c|}{ Occurrence } & \multicolumn{2}{|c|}{ Number } & \multicolumn{2}{|c|}{ Occurrence } & \multicolumn{2}{|c|}{ Number } & & \\
\hline & $\mathrm{n}$ & $\%$ & $\mathrm{n}$ & $\%$ & $\mathrm{n}$ & $\%$ & $\mathrm{n}$ & $\%$ & $Z$ & $\mathrm{p}$ \\
\hline \multicolumn{11}{|l|}{ Histioteuthidae } \\
\hline Histioteuthis atlantica (lower beaks) & 29 & 96.7 & 799 & 15.6 & 28 & 84.8 & 142 & 1.9 & 28.62 & $<0.0001$ \\
\hline Histioteuthis bonnellii corpuscula (lower beaks) & 16 & 53.3 & 21 & 0.4 & 20 & 60.6 & 45 & 0.6 & 1.49 & 0.136 \\
\hline Histioteuthis b. corpuscula/atlantica (upper beaks) & 28 & 93.3 & 720 & 14.1 & 23 & 69.7 & 165 & 2.2 & 25.45 & $<0.0001$ \\
\hline Histioteuthis eltaninae & 27 & 90.0 & 371 & 7.2 & 33 & 100.0 & 1721 & 23.2 & 23.53 & $<0.0001$ \\
\hline Histioteuthis macrohista & 17 & 56.7 & 40 & 0.8 & 5 & 15.2 & 6 & 0.1 & 6.38 & $<0.0001$ \\
\hline Histioteuthis miranda & 20 & 66.7 & 61 & 1.2 & 17 & 51.5 & 49 & 0.7 & 3.14 & 0.002 \\
\hline Histioteuthis sp. & 2 & 6.7 & 2 & $<0.1$ & 3 & 9.1 & 4 & 0.1 & 0.37 & 0.709 \\
\hline Stigmatoteuthis hoylei & 3 & 10.0 & 7 & 0.1 & 2 & 6.1 & 4 & 0.1 & 1.54 & 0.123 \\
\hline \multicolumn{11}{|l|}{ Neoteuthidae } \\
\hline Alluroteuthis antarcticus & 28 & 93.3 & 170 & 3.3 & 30 & 90.9 & 187 & 2.5 & 2.65 & 0.008 \\
\hline Nototeuthis dimegacotyle & 13 & 43.3 & 37 & 0.7 & 8 & 24.2 & 13 & 0.2 & 4.78 & $<0.0001$ \\
\hline \multicolumn{11}{|l|}{ Cycloteuthidae } \\
\hline Cycloteuthis sirventi & 27 & 90.0 & 166 & 3.2 & 20 & 60.6 & 69 & 0.9 & 9.39 & $<0.0001$ \\
\hline Discoteuthis laciniosa & & & & & 2 & 6.1 & 4 & 0.1 & na & na \\
\hline \multicolumn{11}{|l|}{ Mastigoteuthidae } \\
\hline Mastigoteuthis psychrophila & 14 & 46.7 & 30 & 0.6 & 11 & 33.3 & 18 & 0.2 & 3.06 & 0.002 \\
\hline Mastigoteuthis sp. A (Imber) & 3 & 10.0 & 7 & 0.1 & & & & & na & na \\
\hline \multicolumn{11}{|l|}{ Chiroteuthidae } \\
\hline Asperoteuthis lui & 8 & 26.7 & 22 & 0.4 & 20 & 60.6 & 64 & 0.9 & 2.88 & 0.004 \\
\hline Chiroteuthis veranyi & 12 & 40.0 & 32 & 0.6 & 16 & 48.5 & 39 & 0.5 & 0.73 & 0.464 \\
\hline Chiroteuthis sp. F (Imber) & & & & & 4 & 12.1 & 6 & 0.1 & & na \\
\hline \multicolumn{11}{|l|}{ Batoteuthidae } \\
\hline Batoteuthis skolops & 22 & 73.3 & 68 & 1.3 & 30 & 90.9 & 249 & 3.4 & 7.10 & $<0.0001$ \\
\hline \multicolumn{11}{|l|}{ Cranchiidae } \\
\hline Galiteuthis glacialis & 30 & 100.0 & 640 & 12.5 & 33 & 100.0 & 660 & 8.9 & 6.52 & $<0.0001$ \\
\hline Galiteuthis sp. 3 (Imber) & 5 & 16.7 & 7 & 0.1 & 2 & 6.1 & 4 & 0.1 & 1.54 & 0.123 \\
\hline Galiteuthis stC sp. (Imber) & 3 & 10.0 & 6 & 0.1 & & & & & na & na \\
\hline Mesonychoteuthis hamiltoni & 3 & 10.0 & 4 & 0.1 & 4 & 12.1 & 9 & 0.1 & 0.74 & 0.461 \\
\hline Taonius sp. B (Voss) & 18 & 60.0 & 361 & 7.1 & 19 & 57.6 & 125 & 1.7 & 15.31 & $<0.0001$ \\
\hline Taonius sp. (Clarke) & 20 & 66.7 & 101 & 2.0 & 17 & 51.5 & 73 & 1.0 & 4.66 & $<0.0001$ \\
\hline Teuthowenia pellucida & 22 & 73.3 & 52 & 1.0 & 4 & 12.1 & 5 & 0.1 & 7.76 & $<0.0001$ \\
\hline Oegopsida sp. A (Cherel) & & & & & 2 & 6.1 & 3 & $<0.1$ & na & na \\
\hline \multicolumn{11}{|l|}{ Octopoda } \\
\hline \multicolumn{11}{|l|}{ Octopodidae } \\
\hline Undetermined Octopodidae & 7 & 23.3 & 10 & 0.2 & 2 & 6.1 & 4 & 0.1 & 2.33 & 0.020 \\
\hline \multicolumn{11}{|l|}{ Stauroteuthidae } \\
\hline Stauroteuthis gilchristi & & & & & 1 & 3.0 & 2 & $<0.1$ & na & na \\
\hline Cirrata sp. A (Cherel) & & & & & 1 & 3.0 & 2 & $<0.1$ & na & na \\
\hline Unknown uneroded beaks & 4 & 13.3 & 4 & 0.1 & 1 & 3.0 & 1 & $<0.1$ & 1.78 & 0.075 \\
\hline Total & & & 5118 & 100.0 & & & 7422 & 100.0 & & \\
\hline Unidentifiable beaks (eroded) & 19 & 63.3 & 115 & & 23 & 69.7 & 205 & & & \\
\hline
\end{tabular}

(7.1\%), and some very large species like Taningia danae and juveniles of the giant squid Architeuthis dux (Table 3). Comparisons highlighted seasonal variations in squid eaten by wandering albatrosses. The proportions of $K$. longimana and $H$. atlantica increased progressively from June to October (from 5.1 to $13.0 \%$ and from 12.2 to $18.1 \%$; equality of proportion tests [large-sample tests], $Z=6.28$ and 3.92, respectively, both $\mathrm{p}<0.0001$ ), while that of $G$. glacialis decreased (from 19.6 to $7.1 \%, Z=10.42$, p < 0.0001). Two taxa were either absent (Octopoteuthis sp.) or rare (Taonius sp. B [Voss]) in June and August samples but became common $(1.7 \%)$ and main $(12.7 \%)$ items, respectively, in October samples.

\section{Crozet}

Forty-six cephalopod taxa were identified in Crozet food samples. Two main species (>10\%) together accounted for $58.1 \%$ of the total number of beaks, namely $K$. longimana $(35.0 \%)$ and $H$. eltaninae $(23.2 \%)$. Nine common species $(>1 \%)$ together accounted for $36.0 \%$ by number, including G. glacia- 
lis $(8.9 \%)$ and $M$. hyadesi (5.0\%) (Table 3). Marked seasonal variations in the proportions of beaks included a progressive increase in $K$. longimana from June to October (from 12.4 to $48.5 \%, Z=23.02$, p < 0.0001 ), while $H$. eltaninae and $G$. glacialis decreased (from 30.7 to $17.6 \%$ and from 18.3 to $4.4 \%$, $Z=9.96$ and 15.61, respectively, both $\mathrm{p}<0.0001$ ). Taonius sp. B (Voss) was a rare item in June $(0.4 \%)$ and August $(0.6 \%)$, but it became a common squid prey in October (3.1\%). Three samples (1 in June and 2 in August) contained beaks of the ommastrephid Illex argentinus, which is one of the most common bait species used by toothfish longliners.

\section{Comparison between sites}

Wandering albatrosses preyed overall upon the same cephalopod taxa at both localities, but in different proportions. Four oegopsid families accounted for 83.4 and $88.9 \%$ of the total number of cephalopod prey at Kerguelen and Crozet, respectively. Birds from Kerguelen fed more on histioteuthids (39.5 versus $28.8 \%, Z=12.52, \mathrm{p}<0.0001)$, cranchiids $(22.9$ versus $11.8 \%, Z=16.50, \mathrm{p}<0.0001)$, and ommastrephids (6.1 versus $5.2 \%, Z=2.17, \mathrm{p}=0.030$ ), but much less on onychoteuthids (14.9 versus $43.1 \%, Z=$ $33.39, p<0.0001)$ than Crozet albatrosses. At the species level, Kerguelen samples included more beaks of $H$. atlantica and G. glacialis, and fewer beaks of $K$. longimana and $H$. eltaninae than Crozet samples (Table 3).

\section{Prey size}

The range of smallest and largest cephalopods caught by wandering albatrosses were specimens of $H$. macrohista (estimated DML: $5.1 \mathrm{~cm}$, Crozet) and from A. dux (125 cm, Kerguelen), respectively. The lightest and heaviest squid were individuals from Slosarczykovia circumantarctica (estimated body mass: $0.006 \mathrm{~kg}$, Crozet) and again from A. dux (body mass: $45.5 \mathrm{~kg}$, Kerguelen), respectively. Measured LRL from beaks of the same squid species were not statistically different between the 2 localities in 11 species and they were significantly different in 14 species, with cephalopods from Kerguelen being larger in 9 species (Table 4). LRL from the main 4 cephalopods ( $K$. longimana, $H$. atlantica, H. eltaninae, G. glacialis) varied significantly among localities, but the differences were small and their lengthfrequency distributions demonstrate that wandering albatrosses from Kerguelen and Crozet overall preyed upon the same size classes (Figs. 1 \& 2). Those 4 squid species differed greatly in their estimated body masses in the following increasing order: $H$. eltaninae $(0.07 \pm 0.02 \mathrm{~kg}$, Crozet $)<$ G. glacialis $(0.11 \pm 0.01 \mathrm{~kg}$, Kerguelen $)<H$. atlantica $(0.37 \pm$ $0.10 \mathrm{~kg}$, Kerguelen) $<<K$. longimana $(2.17 \pm 0.80$ and $2.69 \pm 1.03 \mathrm{~kg}$ at Crozet and Kerguelen Islands, respectively).

In contrast to cephalopods, only a few dietary remains allowed estimation of fish size. Fish length and body mass averaged $43 \pm 15 \mathrm{~cm}$ and $1.37 \pm 1.68 \mathrm{~kg}$ $(\mathrm{n}=10)$, respectively, with the smallest and largest specimens being 1 icefish Champsocephalus gunnari $(26 \mathrm{~cm} \mathrm{SL}, 131 \mathrm{~g})$ and 1 Patagonian toothfish $(67 \mathrm{~cm}$ $\mathrm{SL}, 4.55 \mathrm{~kg})$, respectively.

\section{Satellite tracking}

Breeding wandering albatrosses were tracked during 17 (9 birds) and 46 (45 birds) foraging trips at Kerguelen and Crozet Islands, respectively. Trip duration $(4.0 \pm 2.9$ and $5.6 \pm 3.8 \mathrm{~d}$, respectively), maximum range from the colony $(569 \pm 423$ and $624 \pm$ $358 \mathrm{~km}$ ) and the total distance covered during a single trip (1984 \pm 1392 and $2522 \pm 1904 \mathrm{~km})$ were not significantly different between Kerguelen and Crozet birds (Mann-Whitney $U=477,462$, and 440, $\mathrm{p}=0.185,0.272$, and 0.453, respectively). Breeding wandering albatrosses from both localities remained essentially at latitudes 42 to $52^{\circ} \mathrm{S}$ that correspond to the Subantarctic Zone sensu lato, between the STF in the north and the PF in the south (Park \& Gamberoni 1997). A few birds crossed the STF and foraged in warmer waters of the Subtropical Zone (Fig. 3). Albatrosses from the 2 localities segregated longitudinally. The smaller number of Kerguelen birds foraged mainly in the vicinity of the archipelago $\left(62-80^{\circ} \mathrm{E}\right)$, while the higher number of Crozet birds showed a wider longitudinal range $\left(40-68^{\circ} \mathrm{E}\right)$, with some individuals foraging in western Kerguelen waters. Consequently, kernel analysis indicated a larger overlap of Crozet birds over the foraging grounds of Kerguelen albatrosses $(0,19$, and $37 \%$ for 50,75 , and $95 \%$ location densities, respectively) than the reverse $(0,11$, and $18 \%$; Fig. 3$)$.

\section{Stable isotopes}

Feather isotopic values of wandering albatross chicks were either not significantly different $\left(\delta^{13} \mathrm{C}\right.$ : 


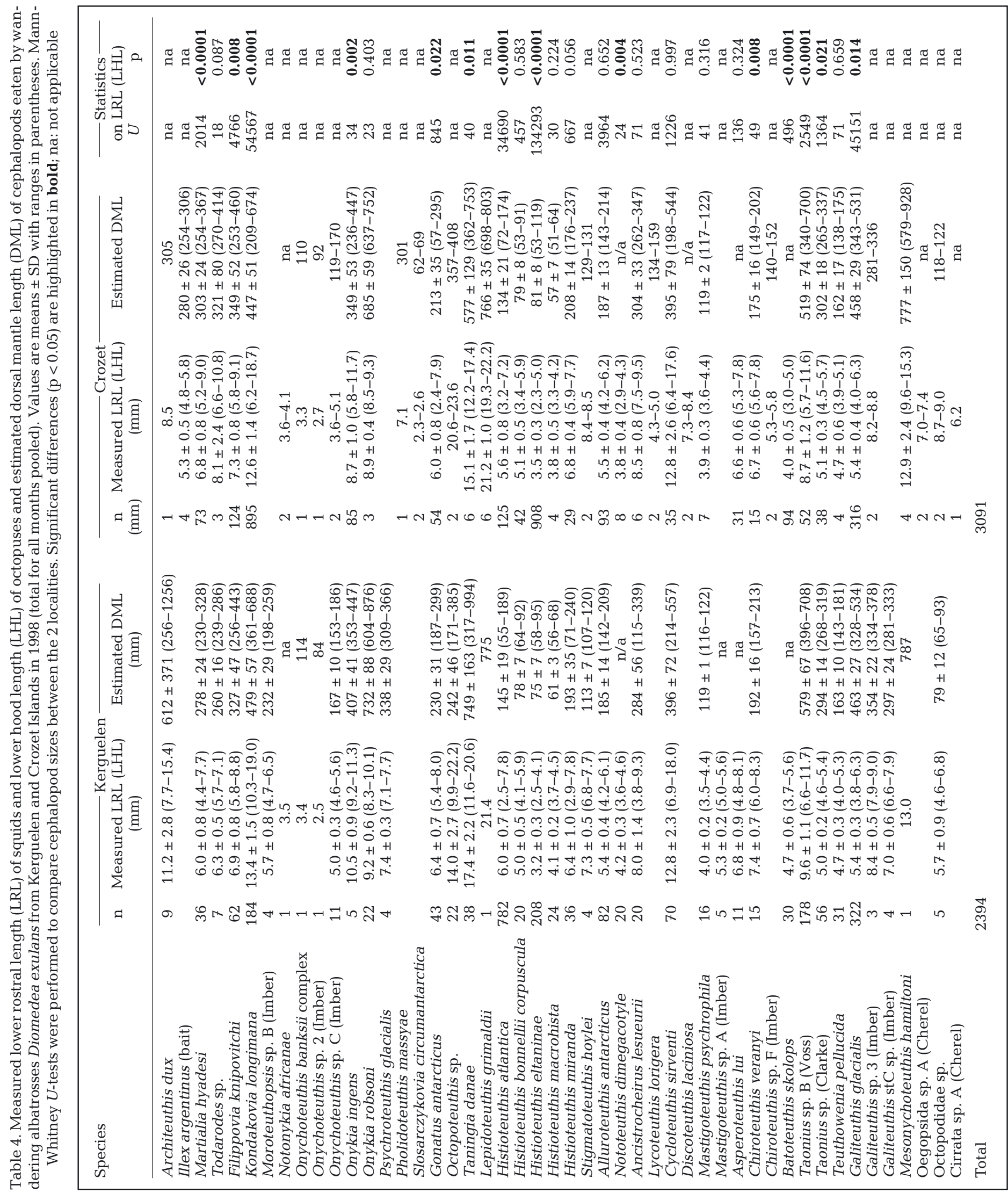




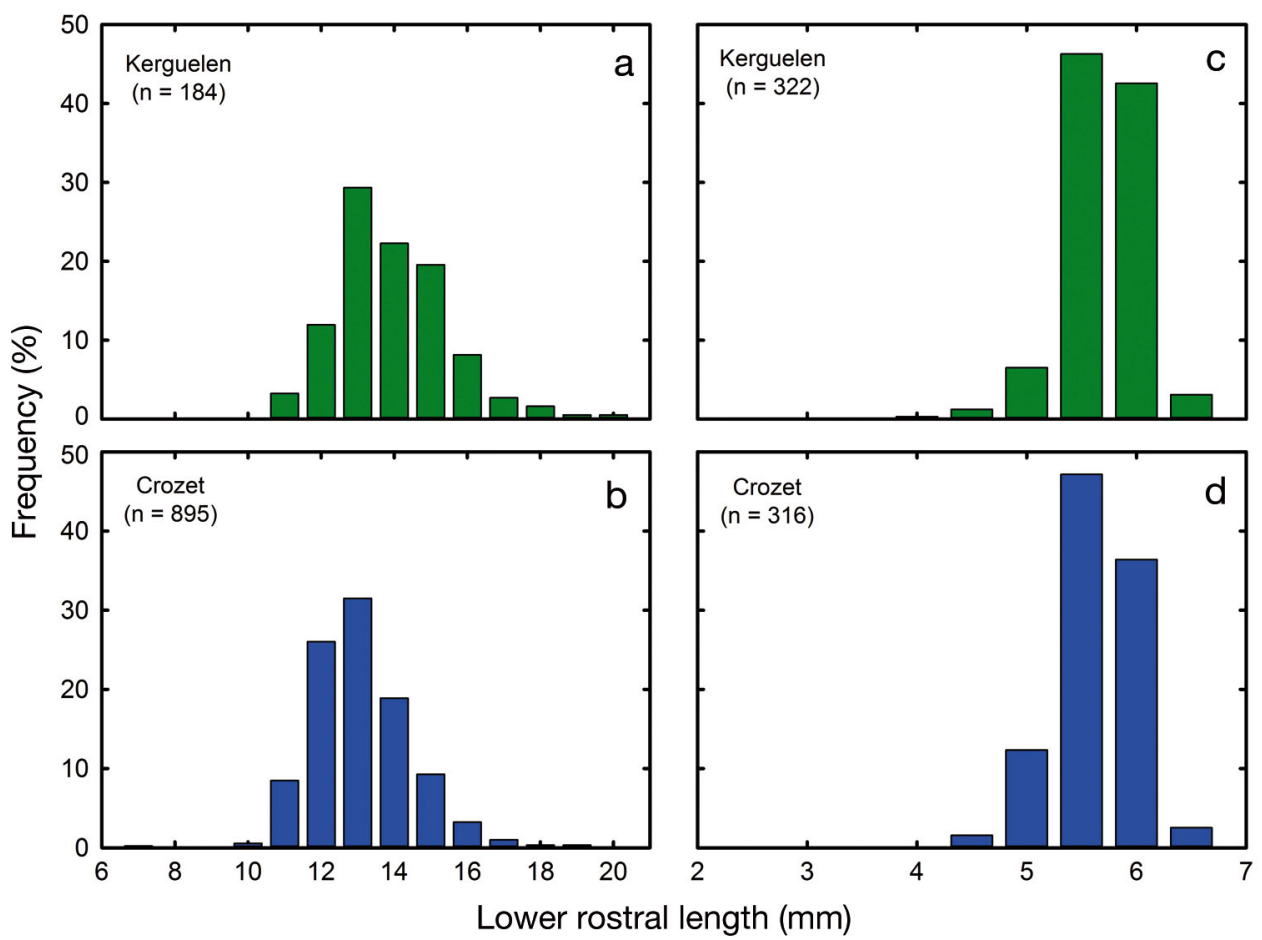

Fig. 1. Length-frequency distribution of lower rostral length of $(\mathrm{a}, \mathrm{b})$ the onychoteuthid squid Kondakovia longimana and $(\mathrm{c}, \mathrm{d})$ the cranchiid squid Galiteuthis glacialis in the diet of wandering albatross Diomedea exulans chicks from (a,c) Kerguelen and $(b, d)$ Crozet Islands

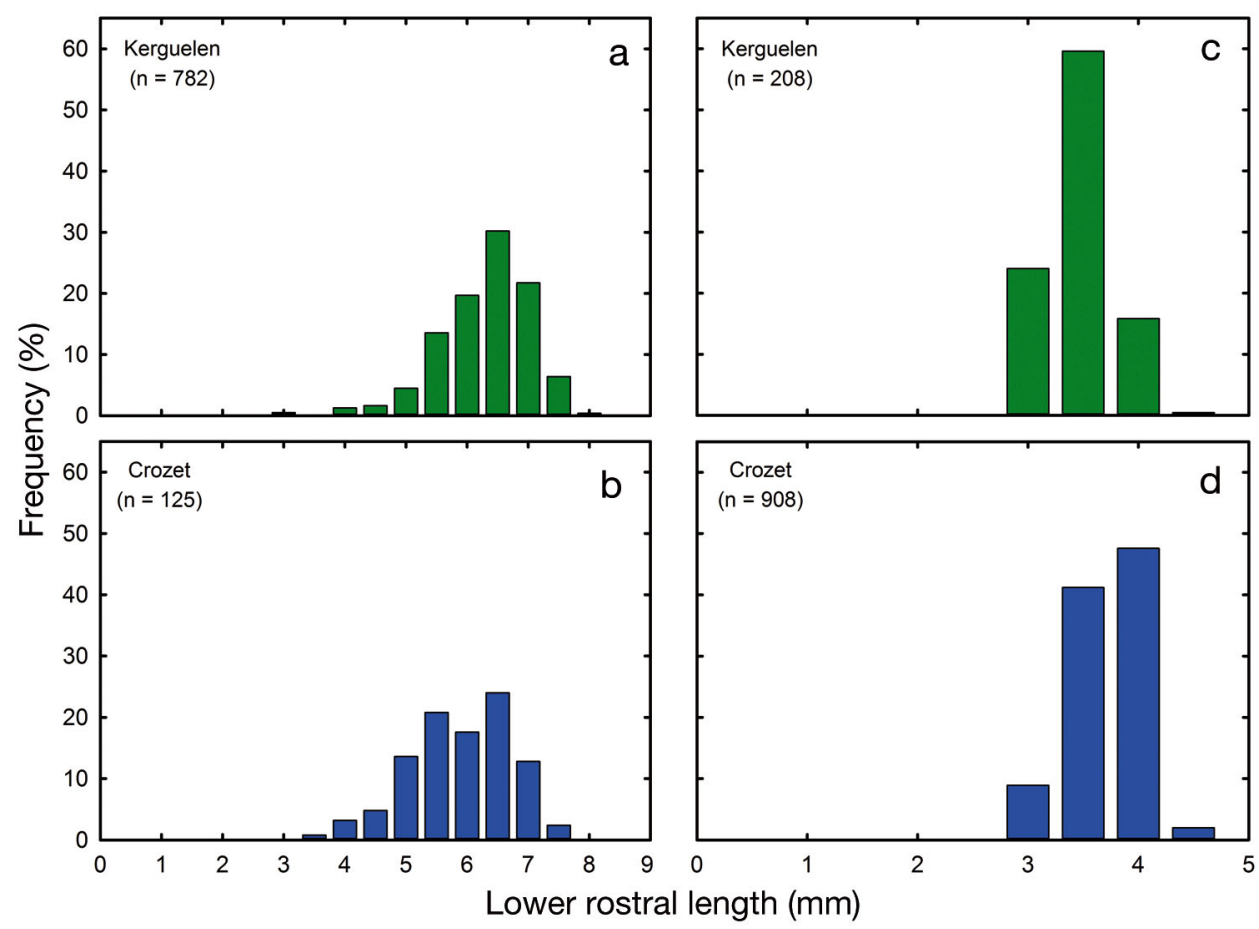

Fig. 2. Length-frequency distribution of lower rostral length of the histioteuthid squids (a,b) Histioteuthis atlantica and (c,d) $H$. eltaninae in the diet of wandering albatross Diomedea exulans chicks from $(\mathrm{a}, \mathrm{c})$ Kerguelen and $(\mathrm{b}, \mathrm{d})$ Crozet Islands 


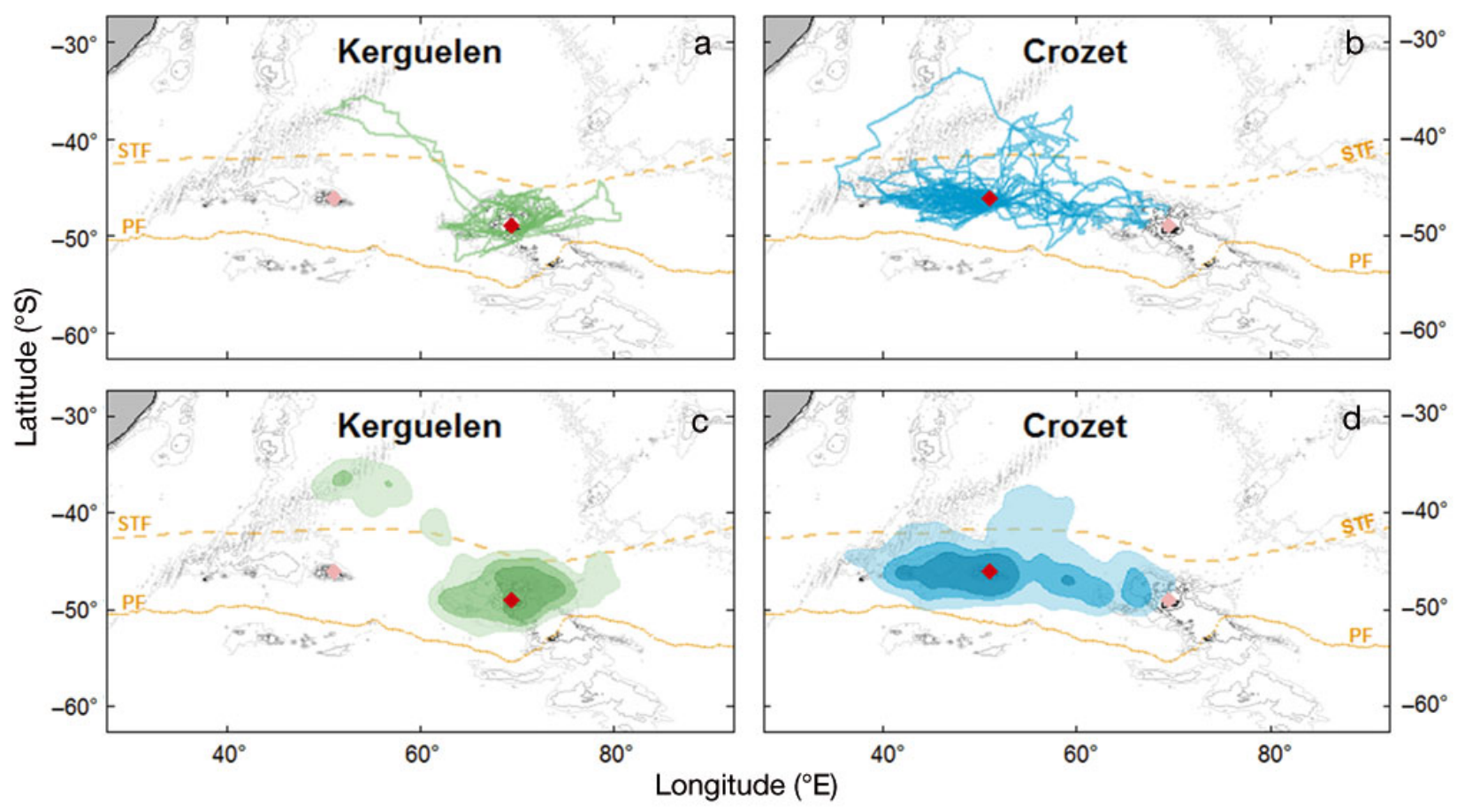

Fig. 3. $(\mathrm{a}, \mathrm{b})$ Satellite tracks and $(\mathrm{c}, \mathrm{d})$ the corresponding density contours resulting from kernel analysis (decreasing darker tone: 50,75 , and $95 \%$ location densities of the core foraging area) of breeding wandering albatrosses Diomedea exulans from $(\mathrm{a}, \mathrm{c})$ Kerguelen and $(\mathrm{b}, \mathrm{d})$ Crozet Islands in 1998. PF: Polar Front; STF: Subtropical Front

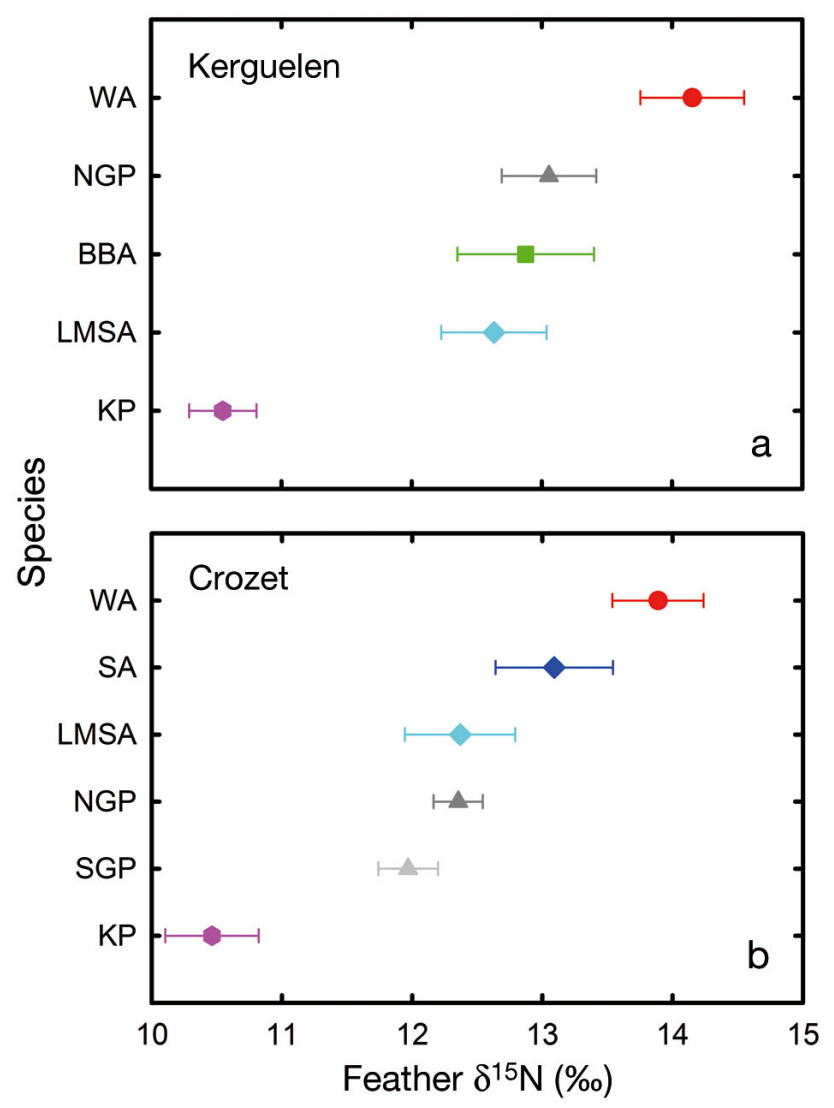

$\mathrm{n}=15$ and $21,-19.3 \pm 0.4$ and $-19.4 \pm 0.4 \%$ at Kerguelen and Crozet Islands, respectively, 2-sample $t$-test, $t=1.12, \mathrm{p}=0.272)$ or marginally significantly different $\left(\delta^{15} \mathrm{~N}: 14.2 \pm 0.4\right.$ and $13.9 \pm 0.3 \%$, respectively, $t=2.13, \mathrm{p}=0.041$ ) between the 2 localities.

Feather isotopic values of albatrosses, giant petrels, and king penguins segregated in both their $\delta^{13} \mathrm{C}$ and $\delta^{15} \mathrm{~N}$ values (ANOVA, Kerguelen: $F_{4,67}=$ 53.68 and 88.48, Crozet: $F_{5,68}=72.84$ and 161.65, respectively, all $\mathrm{p}<0.0001$; Fig. 4). When compared to other large sympatric Procellariiformes and to king penguins, wandering albatross chicks presented higher dorsal body feather $\delta^{15} \mathrm{~N}$ values at both Kerguelen (post hoc Tukey's honestly significant difference tests, all $\mathrm{p} \leq 0.003$ ) and Crozet Islands (all $\mathrm{p}<0.0001$ )

Fig. 4. Feather $\delta^{15} \mathrm{~N}$ values of large Procellariiformes and king penguins Aptenodytes patagonicus (KP) at (a) Kerguelen and (b) Crozet Islands. Species were deliberately placed in a trophic sequence of increasing $\delta^{15} \mathrm{~N}$ values. Values are means \pm SD. BBA: black-browed albatross Thalassarche melanophris; LMSA: light-mantled sooty albatross Phoebetria palpebrata; NGP: northern giant petrel Macronectes halli; SA: sooty albatross $P$. fusca; SGP: southern giant petrel M. giganteus; WA: wandering albatross Diomedea exulans 


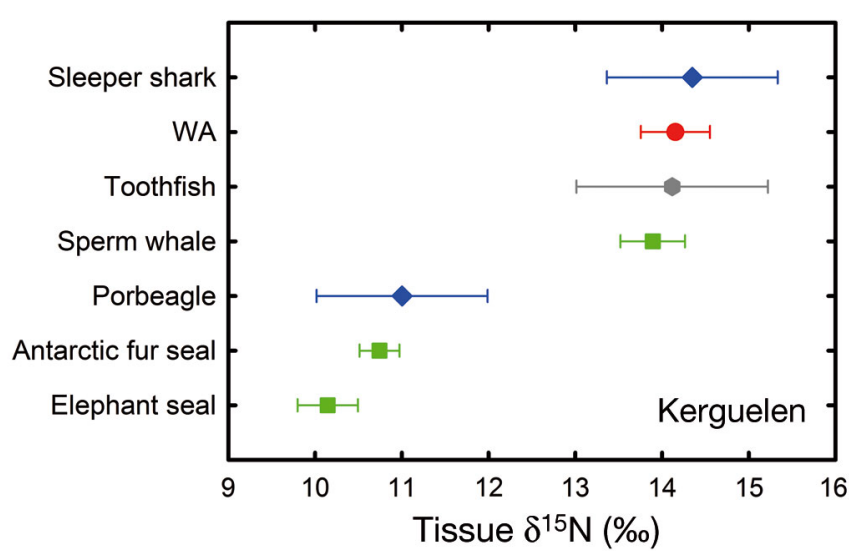

Fig. 5. Tissue $\delta^{15} \mathrm{~N}$ values of predators from Kerguelen Islands. Species were deliberately placed in a trophic sequence of increasing $\delta^{15} \mathrm{~N}$ values. Values are means $\pm \mathrm{SD}$. WA: wandering albatross Diomedea exulans

Tissue $\delta^{15} \mathrm{~N}$ values of wandering albatross and of non-avian predators from the Kerguelen Islands segregated in their $\delta^{15} \mathrm{~N}$ values (Kruskal-Wallis: $H=$ 90.17, p < 0.0001) (Fig. 5). Conover-Inman tests for all pairwise comparisons indicated that the mean $\delta^{15} \mathrm{~N}$ value of wandering albatrosses was higher than $\delta^{15} \mathrm{~N}$ values of elephant seals, Antarctic fur seals, and porbeagle sharks (all $p<0.0001$ ), but it was not significantly different from the values of sperm whales, Patagonian toothfish, and sleeper sharks $(\mathrm{p}=0.566$, 0.757 , and 0.441 , respectively).

\section{DISCUSSION}

Diet

Food of wandering albatross chicks includes primarily fish and cephalopods, with carrion ranking third (reviewed in Table 5). Fish and cephalopods were equally important at the Kerguelen Islands, in agreement with studies conducted at 2 other major breeding sites, South Georgia and Marion Island. In contrast, food composition at the Crozet Archipelago is unique, being consistently dominated by cephalopods in multiple studies over different years. Among other Diomedea albatrosses, a predominance of cephalopods was also found in the 2 species of the neritic southern $D$. epomophora and northern $D$. sanfordi royal albatrosses in the Pacific Ocean. Food mass and composition of wandering albatrosses at the Kerguelen Islands did not present seasonal variations, while significant changes were found at the Crozet Islands. There, the fresh mass of food samples decreased considerably during the late chick-rearing period, with the cephalopod component of the diet dropping by $59 \%$. This, together with a lower feeding rate and declining adult body mass (Weimerskirch \& Lys 2000), suggests either a lowering of feeding conditions at sea from August onwards or that chick provisioning behavior changes as chicks approach fledging mass and adults prepare for flight feather molt.

Table 5. Review of the available information on mass and composition of fresh food from stomach contents of Diomedea albatrosses; nd: no data

\begin{tabular}{|c|c|c|c|c|c|c|c|c|}
\hline Locality & $\begin{array}{l}\text { Sampling } \\
\text { year }\end{array}$ & $\begin{array}{c}\text { Stomach } \\
\text { samples } \\
\text { (n) }\end{array}$ & $\begin{array}{c}\text { Solid mass } \\
\text { mean } \pm \mathrm{SD} \\
(\mathrm{g})\end{array}$ & $\begin{array}{c}\text { Fresh mass } \\
\text { mean } \pm \mathrm{SD}\end{array}$ & $\begin{array}{l}\text { Fish } \\
(\%)\end{array}$ & $\begin{array}{c}\text { Cepha- } \\
\text { lopods } \\
(\%)\end{array}$ & $\begin{array}{l}\text { Others } \\
\text { (\%) }\end{array}$ & Reference \\
\hline \multicolumn{9}{|c|}{ Wandering albatross $D$. exulans } \\
\hline \multirow[t]{5}{*}{ South Georgia } & $1983 \& 1984$ & 79 & nd & nd & 41.5 & 39.5 & 20.0 & Prince \& Morgan (1987) \\
\hline & 1999 & 18 & 426 & nd & 53.1 & 42.1 & 4.8 & Xavier et al. (2004) \\
\hline & 2000 (Mar-Apr) & 9 & nd & nd & 45.6 & 32.0 & 22.3 & Xavier et al. (2003b) \\
\hline & 2000 (May-Aug) & 20 & 630 & nd & 84.3 & 11.3 & 4.4 & Xavier et al. (2004) \\
\hline & 2009 & 35 & nd & nd & 59.4 & 38.4 & 2.2 & Ceia et al. (2012) \\
\hline Marion & 1988-1989 & 50 & $523 \pm 425$ & nd & 36.5 & 58.6 & 4.9 & Cooper et al. (1992) \\
\hline \multirow[t]{4}{*}{ Crozet } & 1982 & 37 & nd & $297 \pm 339$ & 14.9 & 76.7 & 8.4 & Ridoux (1994) \\
\hline & 1992 & 24 & $511 \pm 243$ & $427 \pm 216$ & 24.3 & 72.4 & 3.3 & Weimerskirch et al. (1997b) \\
\hline & 1994 & 10 & nd & $623 \pm 336$ & 10.7 & 75.9 & 13.4 & $\begin{array}{l}\text { Cherel \& Weimerskirch } \\
\text { (1999, unpubl. data) }\end{array}$ \\
\hline & 1998 & 33 & $673 \pm 322$ & $569 \pm 325$ & 11.9 & 87.4 & 0.7 & This study \\
\hline Kerguelen & 1998 & 30 & $388 \pm 185$ & $334 \pm 181$ & 48.3 & 45.6 & 6.1 & This study \\
\hline \multicolumn{9}{|c|}{ Northern royal albatross $D$. sanfordi } \\
\hline Chatham & nd & nd & nd & nd & 14 & 85 & 1 & Marchant \& Higgins (1990) \\
\hline New Zealand & nd & nd & nd & nd & 15 & 80 & 5 & Marchant \& Higgins (1990) \\
\hline \multicolumn{9}{|c|}{ Southern royal albatross $D$. epomophora } \\
\hline Campbell & nd & nd & nd & nd & 21 & 75 & 4 & Marchant \& Higgins (1990) \\
\hline
\end{tabular}


Fish prey of wandering albatrosses is difficult to identify due to their low number and digestion state. However, 2 consistent features are notable: (1) individual fishes are of medium to large size $(>10 \mathrm{~cm})$, and (2) most of them belong to deep-water benthopelagic species (Croxall et al. 1988, Cooper et al. 1992, Xavier et al. 2003b, 2004, Ceia et al. 2012, this study). The prevalence of deep-sea fish is also indicated by the occurrence of their associated large female parasitic copepods in food samples (Ridoux 1994, this study). How albatrosses catch deep-sea fish remains unclear (Croxall et al. 1988, Cherel et al. 2000). Some are undoubtedly eaten in association with fishing vessels (Nel et al. 2002), but they were already natural prey of albatrosses before development of fisheries in the Southern Ocean (Ridoux 1994, Weimerskirch et al. 1997b). Wandering albatrosses are surface-feeders and scavengers (Prince \& Morgan 1987, Cherel \& Klages 1998). They thus probably feed on dead, dying, or incapacitated fish that rise to the surface, which is the most likely explanation for the abundance of deep-sea squids in their diet (Nesis et al. 1998, Cherel \& Weimerskirch 1999, Xavier \& Croxall 2007).

Diomedea albatrosses are the seabirds that prey upon the largest diversity of cephalopods (Imber 1992, Croxall \& Prince 1996). Accumulated beaks from food samples of wandering albatross from Kerguelen Islands confirm this typical feature. Overall, wandering albatrosses from different localities fed on the same cephalopod species, but in different proportions. A dietary review highlights 6 main characteristics of cephalopods eaten by Diomedea albatrosses (Table 6). First,

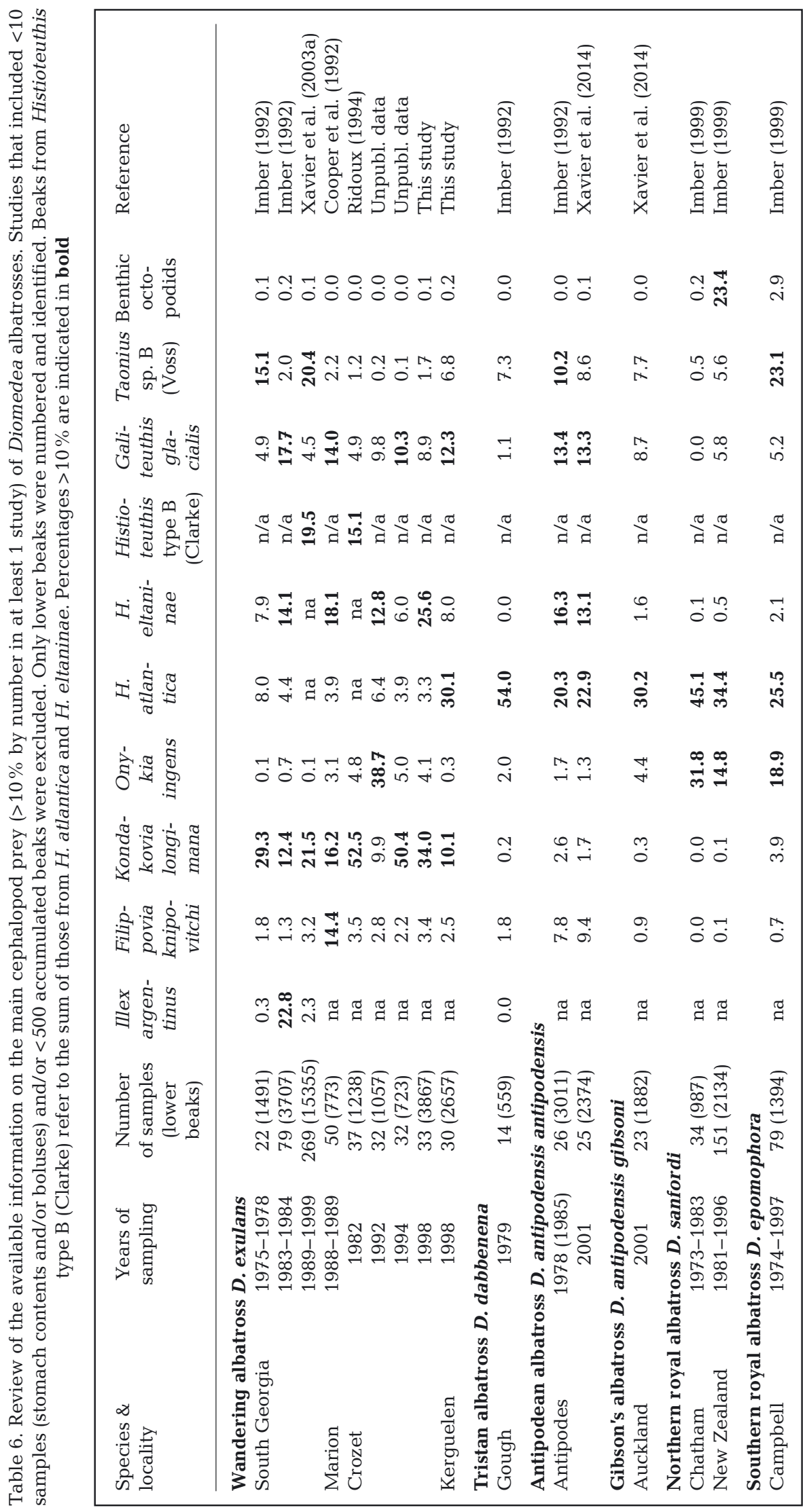


birds feed almost exclusively on adult squids, as indicated by both the wholly darkened beaks and the corresponding estimated body sizes of cephalopods (Cherel \& Weimerskirch 1999, Xavier et al. 2003a). Second, albatrosses target oceanic squids (Cherel \& Klages 1998), with the exception of the northern royal albatrosses that prey significantly on benthic octopodids over the New Zealand shelf (Imber 1999). Third, 3 families dominated the squid diet, namely Onychoteuthidae, Histioteuthidae, and Cranchiidae. The same 3 families constitute a significant part of the food of cetaceans, including the sperm whale Physeter macrocephalus, in the Southern Ocean (Clarke 1980, 1996). Fourth, Diomedea species forage on several species of histioteuthids, with Histioteuthis eltaninae being generally the main species in southern waters and $H$. atlantica farther north. The only latitudinal exception is the predominance of $H$. atlantica at Kerguelen Islands (this study). Fifth, while Tristan, Antipodean D. antipodensis antipodensis, and Gibson's D. a. gibsoni albatrosses do not prey significantly on onychoteuthids, wandering and royal albatrosses target primarily Kondakovia longimana and Onykia ingens, respectively, which, owing to their large size, form a major part of the birds' diet by mass (Cooper et al. 1992, Imber 1999, Xavier et al. 2003a). Sixth, the southernmost Diomedea species prey upon large numbers of Galiteuthis glacialis and/or Taonius sp. B (Voss), while Tristan and northern royal albatrosses do not feed significantly on cranchiids (Table 6).

Seasonal dietary changes of wandering albatrosses included consistent variations in the proportions of some squid beaks in both Kerguelen and Crozet food samples. Three species illustrate well those temporal variations and the usefulness of cephalopod consumers as biological samplers of poorly known oceanic squids (Cherel \& Weimerskirch 1999). The proportion of beaks of G. glacialis decreased over time, those of $K$. longimana increased, while beaks of Taonius sp. B (Voss) occurred in significant numbers in October samples only. Beaks were from adult squids, and post-spawning moribund or dead cranchiids and onychoteuthids are known to rise to and float at the sea surface where they become available for the birds (Nesis et al. 1998, Lynnes \& Rodhouse 2002). Our study confirms that $K$. longimana reproduces during the winter months not only in Crozet (Cherel \& Weimerskirch 1999) but also in Kerguelen waters. Our results also indicate that $G$. glacialis spawns in fall and early winter, and Taonius sp. B (Voss) in late winter in the southern Indian Ocean.

\section{Foraging zones and habitat}

Biogeography of fresh prey indicates that adults fed their chicks with cephalopods and fishes caught in slope and oceanic waters surrounding the Kerguelen and Crozet Islands (Cherel et al. 2004, 2011, Duhamel et al. 2005). Feeding primarily in subantarctic waters is also supported by feather isotopic values, with all chick $\delta^{13} \mathrm{C}$ values clustering from -20.3 to $-18.5 \%$, i.e. between the estimated isotopic positions of the PF and STF (Jaeger et al. 2010). Indeed, satellite-tracked birds in late incubation and during the brooding period in 1998 foraged mainly in a radius of $\sim 600 \mathrm{~km}$ around the archipelagoes. Wandering albatrosses from Kerguelen segregated at sea from Crozet birds by favoring eastern Kerguelen waters, while Crozet albatrosses primarily foraged in western Crozet waters (Fig. 4). Some Crozet birds foraged off western Kerguelen, thus inducing an overlap between the foraging grounds of wandering albatrosses from the 2 archipelagoes (Weimerskirch 1998, Weimerskirch et al. 2015, this study).

A close examination of accumulated beaks highlights 2 relevant features about the latitudinal feeding zones of wandering albatrosses during the winter months. (1) The near lack of beaks from the endemic Antarctic species Psychroteuthis glacialis (Collins \& Rodhouse 2006) precludes birds feeding significantly at high latitudes in winter. This is in agreement with satellite tracking and isotopic investigations during incubation at the Crozet Islands showing that only old males forage in Antarctica, while younger males and females favor subantarctic and subtropical waters, respectively (Lecomte et al. 2010, Jaeger et al. 2014, Weimerskirch et al. 2014). (2) Some cephalopods were temperate and warmwater species (Ancistrocheirus lesueurii, H. bonnellii corpuscula, H. miranda; Voss et al. 1998, Young et al. 2016), thus indicating foraging north of the STF. There is thus a mismatch between fresh items that were caught locally and some accumulated items that were taken farther away in warmer waters. The mismatch is easily explained by breeding wandering albatrosses performing long looping trips during which they first feed for themselves, with the resulting accumulated beaks being regurgitated to the chicks together with fresh prey that are caught either on the way back to the colonies or during shorter commuting trips to nearby waters (Weimerskirch et al. 1997b, Weimerskirch 1998). A main and expected conclusion of satellite tracking, prey biogeography, and stable isotopes is that wandering albatrosses from Kerguelen behave at sea 
essentially as those from Crozet and Marion Islands during the chick-rearing period, with dietary differences being likely related to the local environments, such as the predominance of $H$. atlantica over $H$. eltaninae in Kerguelen waters and the reverse in Crozet waters (Table 3; Cherel et al. 2004).

\section{Wandering albatross as an apex predator/scavenger}

At both Kerguelen and Crozet Islands, wandering albatrosses had higher dorsal body feather $\delta^{15} \mathrm{~N}$ values than smaller albatrosses and giant petrels, the major Southern Ocean seabird scavengers. Identical isotopic results were found in South Georgia, where the wandering albatross dominates over the northern and southern giant petrels and over the blackbrowed, grey-headed, and light-mantled sooty albatrosses (Y. Cherel \& R. A. Phillips unpubl. data). Hence, whatever the breeding locality, the wandering albatross is the species with the highest trophic position within the communities of oceanic seabirds, which also include many species of meso-predators, such as penguins and smaller Procellariiformes (Blévin et al. 2013).

Tissue $\delta^{15} \mathrm{~N}$ comparison amongst various predators from the Kerguelen Islands highlighted the high trophic position of the wandering albatross. The species showed higher $\delta^{15} \mathrm{~N}$ values than the king penguin, other myctophid eaters (elephant seal and Antarctic fur seal), and the porbeagle shark that feeds on both micronektonic fish and juvenile squids (Lea et al. 2002, Cherel \& Duhamel 2004, Cherel et al. 2008). In contrast, the high $\delta^{15} \mathrm{~N}$ value of wandering albatross clustered with those from 3 large to very large predators, the Patagonian toothfish, sleeper shark, and sperm whale. Wandering albatross, toothfish, and sleeper shark are opportunistic predators and scavengers (Cherel \& Duhamel 2004, Cherel et al. 2004), while sperm whales target primarily adult squids (Mikhalev et al. 1981). Together with the colossal squid Mesonychoteuthis hamiltoni (Cherel et al. 2008), the 4 species thus constitute the apex consumers of the pelagic ecosystem in Kerguelen waters.

\section{Relationships with fisheries}

Anthropogenic items were found in half of the food samples collected in 1998. They included plastic fragments, bait remains, and hooks/snoods. Plastic pieces are common in stomach contents of seabirds (Wilcox et al. 2015), and they are more frequent in Diomedea species than in smaller albatrosses (Jiménez et al. 2015). Wandering albatross chicks from Kerguelen and Crozet Islands contained low plastic loads in their stomach, which contrasts with the higher loads of albatross chicks from the North Pacific (Auman et al. 1998, Young et al. 2009) and minimizes potential deleterious mechanical and physiological effects from plastic consumption.

Bait used on toothfish longlines includes fish and squids that do not occur naturally in the southern Indian Ocean. Accordingly, sardine Sardinops sp. and Illex argentinus have been identified in the diet of the commonest seabird attracted by fishing vessels, the white-chinned petrel Procellaria aequinoctialis, from Crozet and Kerguelen Islands (Catard et al. 2000, Delord et al. 2010). The presence of beaks of $I$. argentinus in the present work adds the wandering albatross to the list of seabirds that consume baits and are thus at risk of being directly killed by hooks during longline operations. Another, more indirect risk for wandering albatross is related to the presence of severely or completely corroded hooks in their stomachs (Nel \& Nel 1999, Phillips et al. 2010, this study). Their vulture-like hyperacidic gastric fluids (Grémillet et al. 2012) digest hooks that could lead to the absorption of harmful heavy metals whose effects on the birds remain to be determined.

The amount of fishing gear found in association with the wandering albatross is an order of magnitude greater than in other Procellariiformes (Phillips et al. 2010). The strong relationship between fisheries and the species is exemplified by the presence of a large number of hooks and snoods in Crozet food samples. As reported elsewhere ( $\mathrm{Nel} \& \mathrm{Nel}$ 1999, Phillips et al. 2010), all hooks and snoods were from the local Patagonian toothfish fishery that uses an automatic baited system, and not from tuna industry located farther north that uses larger hooks. Its wide gape allows the wandering albatross to swallow large prey (Phillips et al. 2010). Hence, the species scavenges on sizeable discarded nontarget fish and toothfish heads that are rejected during processing and dumped without the hooks being removed, a procedure that is forbidden by Commission for the Conservation of Antarctic Marine Living Resources (CCAMLR) fishery regulations (Nel \& Nel 1999, Phillips et al. 2010). Only 1 legal longliner (Crozet), and 2 legal trawlers plus a single longliner (Kerguelen) operated in the area during the Austral winter 1998. At that time, 5 illegal longliners were arrested in Kerguelen waters by 
the French Navy, which did not patrol Crozet waters. Moreover, landing of Patagonian toothfish indicated that at least 8 illegal longliners fished around Kerguelen and 3, possibly as many as 15, operated in Crozet waters in winter 1998 (G. Duhamel pers. comm.). Stomach hooks from Crozet birds indirectly confirm the presence of a fairly large number of illegal vessels in the area during the study period. This timing agrees with the beginning of considerable poaching in 1996 that increased quickly to unsustainably high levels during the subsequent years in both the westernmost Marion Island and easternmost Kerguelen Archipelago (Nel \& Nel 1999, Lord et al. 2006). Remarkably, the composition of wandering albatross food remained apparently unaffected by the presence of fishing vessels, with squid consistently dominating the chick diet at the Crozet Islands (Ridoux 1994, Cherel \& Weimerskirch 1999, this study).

\section{Perspectives}

Since Diomedea albatrosses are threatened by interactions with commercial fisheries and environmental changes (Barbraud et al. 2012), understanding their prey requirements and dietary flexibility is important for their effective conservation and management. A review of Diomedea feeding habits pinpoints the need for more direct dietary investigations on this endangered group of apex marine predators. Four features are notable: (1) only anecdotal information is available during the most inaccessible phases of albatross life, in early years at sea and during the inter-breeding period; (2) most stomach sample studies focused on chick diet, because birds carry no food in their stomach when they land during other breeding stages; (3) composition of the chick food is well-described only for the wandering albatross (Table 5); (4) owing to the large number of accumulated beaks, cephalopod prey is detailed in all but 1 taxa (Table 6); (5) nothing is known about the feeding habits of the Amsterdam albatross, the rarest and most localized Diomedea species. As a starting point, we consequently recommend: (1) to collect food samples from recently fed large chicks to determine fresh prey composition, species, and size; (2) to validate on chicks the promising and non-invasive DNA-based dietary analysis of feces (Bowser et al. 2013); and (3) to generalize the latter approach on adults during different breeding stages, and on non-breeding adults and immature albatrosses when present on land.
Acknowledgements. We thank S. Caulle, T. Guionnet, and D. Secondi, who helped during the field work; G. Guillou (LIENSs, La Rochelle) for stable isotope analysis; and G. Duhamel (MNHN, Paris) for providing information about the legal and illegal fisheries in Kerguelen and Crozet waters during the study period. This work was supported financially and logistically by the Institut Polaire Français Paul Emile Victor (Programme No. 109, H.W.) and the Terres Australes et Antarctiques Françaises.

\section{LITERATURE CITED}

Andersen NC (1984) Genera and subfamilies of the family Nototheniidae (Pisces, Perciformes) from the Antarctic and Subantarctic. Steenstrupia (Cph) 10:1-34

Auman HJ, Ludwig JP, Giesy JP, Colborn T (1998) Plastic ingestion by Laysan albatross chicks on Sand Island, Midway Atoll, in 1994 and 1995. In: Robertson G, Gales R (eds) Albatross biology and conservation. Surrey Beatty and Sons, Chipping Norton, p 239-244

* Barbraud C, Rolland V, Jenouvrier S, Nevoux M, Delord K, Weimerskirch H (2012) Effects of climate change and fisheries bycatch on Southern Ocean seabirds: a review. Mar Ecol Prog Ser 454:285-307

* Belkin IM, Gordon AL (1996) Southern Ocean fronts from the Greenwich meridian to Tasmania. J Geophys Res 101:3675-3696

Blévin P, Carravieri A, Jaeger A, Chastel O, Bustamante P, Cherel Y (2013) Wide range of mercury contamination in chicks of Southern Ocean seabirds. PLOS ONE 8:e54508

Bowser AK, Diamond AW, Addison JA (2013) From puffins to plankton: a DNA-based analysis of a seabird food chain in the northern Gulf of Maine. PLOS ONE 8: e83152

* Brothers N, Gales R, Hedd A, Robertson G (1998) Foraging movements of the shy albatross Diomedea cauta breeding in Australia; implications for interactions with longline fisheries. Ibis 140:446-457

Calenge C (2006) The package 'adehabitat' for the R software: a tool for the analysis of space and habitat use by animals. Ecol Model 197:516-519

Carravieri A, Bustamante P, Churlaud C, Fromant A, Cherel Y (2014) Moulting patterns drive within-individual variations of stable isotopes and mercury in seabird body feathers: implications for monitoring of the marine environment. Mar Biol 161:963-968

* Catard A, Weimerskirch H, Cherel Y (2000) Exploitation of distant Antarctic waters and close shelf-break waters by white-chinned petrels rearing chicks. Mar Ecol Prog Ser 194:249-261

*eia FR, Phillips RA, Ramos JA, Cherel Y, Vieira RP, Richard P, Xavier JC (2012) Short- and long-term consistency in the foraging niche of wandering albatrosses. Mar Biol 159:1581-1591

* Cherel Y, Duhamel G (2004) Antarctic jaws: cephalopod prey of sharks in Kerguelen waters. Deep-Sea Res I 51: $17-31$

Cherel Y, Hobson KA (2007) Geographical variation in carbon stable isotope signatures of marine predators: a tool to investigate their foraging areas in the Southern Ocean. Mar Ecol Prog Ser 329:281-287

Cherel Y, Klages N (1998) A review of the food of albatrosses. In: Robertson G, Gales R (eds) Albatross biology and conservation. Surrey Beatty and Sons, Chipping Norton, p 113-136

Cherel Y, Weimerskirch H (1999) Spawning cycle of ony- 
choteuthid squids in the southern Indian Ocean: new information from seabird predators. Mar Ecol Prog Ser 188:93-104

Cherel Y, Weimerskirch H, Duhamel G (1996) Interactions between longline vessels and seabirds in Kerguelen waters and a method to reduce seabird mortality. Biol Conserv 75:63-70

* Cherel Y, Weimerskirch H, Trouvé C (2000) Food and feeding ecology of the neritic-slope forager black-browed albatross and its relationships with commercial fisheries in Kerguelen waters. Mar Ecol Prog Ser 207:183-199

K Cherel Y, Duhamel G, Gasco N (2004) Cephalopod fauna of subantarctic islands: new information from predators. Mar Ecol Prog Ser 266:143-156

* Cherel Y, Ducatez S, Fontaine C, Richard P, Guinet C (2008) Stable isotopes reveal the trophic position and mesopelagic fish diet of female southern elephant seals breeding on the Kerguelen Islands. Mar Ecol Prog Ser 370: 239-247

Cherel Y, Fontaine C, Richard P, Labat JP (2010) Isotopic niches and trophic levels of myctophid fishes and their predators in the Southern Ocean. Limnol Oceanogr 55: 324-332

Cherel Y, Gasco N, Duhamel G (2011) Top predators and stable isotopes document the cephalopod fauna and its trophic relationships in Kerguelen waters. In: Duhamel G, Welsford D (eds) The Kerguelen Plateau: marine ecosystem and fisheries. Société Française d'Ichtyologie, Paris, p 99-108

Clarke MR (1980) Cephalopoda in the diet of sperm whales of the Southern Hemisphere and their bearing on sperm whale biology. Discov Rep 37:1-324

Clarke MR (1986) A handbook for the identification of cephalopod beaks. Clarendon Press, Oxford

* Clarke MR (1996) Cephalopods as prey. III. Cetaceans. Philos Trans R Soc Lond B Biol Sci 351:1053-1065

Collins MA, Rodhouse PGK (2006) Southern Ocean cephalopods. Adv Mar Biol 50:191-265

Cooper J, Henley SR, Klages NTW (1992) The diet of the wandering albatross Diomedea exulans at sub-antarctic Marion Island. Polar Biol 12:477-484

* Croxall JP, Prince PA (1996) Cephalopods as prey. I. Seabirds. Philos Trans R Soc Lond B Biol Sci 351:1023-1043

Croxall JP, North AW, Prince PA (1988) Fish prey of the wandering albatross Diomedea exulans at South Georgia. Polar Biol 9:9-16

de Solla SR, Bonduriansky R, Brooks RJ (1999) Eliminating autocorrelation reduces biological relevance of home range estimates. J Anim Ecol 68:221-234

Delord K, Cotté C, Péron C, Marteau C and others (2010) Atsea distribution and diet of an endangered top predator: relationship between white-chinned petrels and commercial longline fisheries. Endang Species Res 13:1-16

Duhamel G, Gasco P, Davaine P (2005) Poissons des Iles Kerguelen et Crozet. Guide régional de l'océan Austral. Muséum national d'Histoire naturelle, Paris

Gallerani Lawson EJ, Rodgers AR (1997) Differences in home-range size computed in commonly used software programs. Wildl Soc Bull 25:721-729

Grémillet D, Prudor A, Le Maho Y, Weimerskirch H (2012) Vultures of the seas: hyperacidic stomachs in wandering albatrosses as an adaptation to dispersed food resources, including fishery wastes. PLOS ONE 7:e37834

Imber MJ (1992) Cephalopods eaten by wandering albatrosses (Diomedea exulans L.) breeding at six circumpolar localities. J R Soc NZ 22:243-263

Imber MJ (1999) Diet and feeding ecology of the royal alba- tross Diomedea epomophora, king of the shelf break and inner slope. Emu 99:200-211

Jaeger A, Cherel Y (2011) Isotopic investigation of contemporary and historic changes in penguin trophic niches and carrying capacity of the Southern Indian Ocean. PLOS ONE 6:e16484

Jaeger A, Lecomte VJ, Weimerskirch H, Richard P, Cherel Y (2010) Seabird satellite tracking validates the use of latitudinal isoscapes to depict predators' foraging areas in the Southern Ocean. Rapid Commun Mass Spectrom 24: 3456-3460

Jaeger A, Jaquemet S, Phillips RA, Wanless RM, Richard P, Cherel Y (2013) Stable isotopes document inter- and intra-specific variation in feeding ecology of nine large southern Procellariiformes. Mar Ecol Prog Ser 490: 255-266

Jaeger A, Goutte A, Lecomte VJ, Richard P and others (2014) Age, sex and breeding status shape a complex foraging pattern in an extremely long-lived seabird. Ecology 95:2324-2333

Jiménez S, Domingo A, Brazeiro A, Defeo O, Phillips RA (2015) Marine debris ingestion by albatrosses in the southwest Atlantic Ocean. Mar Pollut Bull 96:149-154

Jouventin P, Weimerskirch H (1990) Satellite tracking of wandering albatrosses. Nature 343:746-748

Lea MA, Cherel Y, Guinet C, Nichols PD (2002) Antarctic fur seals foraging in the Polar Frontal Zone: inter-annual shifts in diet as shown from fecal and fatty acid analyses. Mar Ecol Prog Ser 245:281-297 (Erratum in Mar Ecol Prog Ser 253:310, 2003)

K Lecomte VJ, Sorci G, Cornet S, Jaeger A and others (2010) Patterns of aging in the long-lived wandering albatross. Proc Natl Acad Sci USA 107:6370-6375

Lord C, Duhamel G, Pruvost P (2006) The Patagonian toothfish (Dissostichus eleginoides) fishery in the Kerguelen Islands (Indian Ocean sector of the Southern Ocean). CCAMLR Sci 13:1-25

Lu CC, Ickeringill R (2002) Cephalopod beak identification and biomass estimation techniques: tools for dietary studies of southern Australian finfishes. Mus Vic Sci Rep 6:1-65

Lynnes AS, Rodhouse PG (2002) A big mouthful for predators: the largest recorded specimen of Kondakovia longimana (Cephalopoda: Onychoteuthidae). Bull Mar Sci 71:1087-1090

Marchant S, Higgins PJ (1990) Handbook of Australian, New Zealand and Antarctic Birds, Vol 1. Oxford University Press, Melbourne

* McConnell BJ, Chambers C, Fedak MA (1992) Foraging ecology of southern elephant seals in relation to the bathymetry and productivity of the Southern Ocean. Antarct Sci 4:393-398

Mikhalev JA, Savusin VP, Kishiyan NA (1981) To the problem of the feeding of sperm whales from the Southern Hemisphere. Rep Int Whaling Comm 31:737-745

Murano M (1999) Mysidacea. In: Boltovskoy D (ed) South Atlantic zooplankton. Backhuys Publishers, Leiden, p 1099-1140

Nel DC, Nel JL (1999) Marine debris and fishing gear associated with seabirds at sub-Antarctic Marion Island, 1996/97 and 1997/98: in relation to longline fishing activity. CCAMLR Sci 6:85-96

Nel DC, Ryan PG, Nel JL, Klages NTW, Wilson RP, Robertson G, Tuck GN (2002) Foraging interactions between wandering albatrosses Diomedea exulans breeding on Marion Island and long-line fisheries in the southern Indian Ocean. Ibis 144:E141-E154 
Nesis KN, Nigmatullin CM, Nikitina IV (1998) Spent females of deepwater squid Galiteuthis glacialis under the ice at the surface of the Weddell Sea (Antarctica). J Zool (Lond) 244:185-200

Park YH, Gamberoni L (1997) Cross-frontal exchange of Antarctic intermediate water and Antarctic bottom water in the Crozet Basin. Deep-Sea Res II 44:963-986

Phillips RA (2006) Efficacy and effects of diet sampling of albatross chicks. Emu 106:305-308

Phillips RA, Hamer KC (2000) Postnatal development of northern fulmar chicks, Fulmarus glacialis. Physiol Biochem Zool 73:597-604

Phillips RA, Xavier JC, Croxall JP (2003) Effects of satellite transmitters on albatrosses and petrels. Auk 120: 1082-1090

* Phillips RA, Ridley C, Reid K, Pugh PJA, Tuck GN, Harrison N (2010) Ingestion of fishing gear and entanglements of seabirds: monitoring and implications for management. Biol Conserv 143:501-512

Prince PA, Morgan RA (1987) Diet and feeding ecology of Procellariiformes. In: Croxall JP (ed) Seabirds. Feeding ecology and role in marine ecosystems. Cambridge University Press, Cambridge, p 135-171

Prince PA, Wood AG, Barton T, Croxall JP (1992) Satellite tracking of wandering albatrosses (Diomedea exulans) in the South Atlantic. Antarct Sci 4:31-36

Ridoux V (1994) The diets and dietary segregation of seabirds at the subantarctic Crozet Islands. Mar Ornithol 22: $1-92$

Sallee JB, Speer K, Morrow R (2008) Southern Ocean fronts and their variability to climate modes. J Clim 21: 3020-3039

Smale MJ, Watson G, Hecht T (1995) Otolith atlas of Southern African marine fishes. JLB Smith Institute of Ichthyology, Grahamstown

Vanderklift MA, Ponsard S (2003) Sources of variation in consumer-diet $\delta^{15} \mathrm{~N}$ enrichment: a meta-analysis. Oecologia 136:169-182

Voss NA, Nesis KN, Rodhouse PG (1998) The cephalopod Family Histioteuthidae (Oegopsida): systematics, biology, and biogeography. Smithson Contrib Zool 586:293-372

Weimerskirch H (1998) Foraging strategies of Indian Ocean albatrosses and their relationships with fisheries. In: Robertson G, Gales R (eds) Albatross biology and conservation. Surrey Beatty and Sons, Chipping Norton, p 168-179

Weimerskirch H, Lys P (2000) Seasonal changes in the provisioning behaviour and mass of male and female wandering albatrosses in relation to the growth of the chicks. Polar Biol 23:733-744

Weimerskirch H, Salamolard M, Sarrazin F, Jouventin P (1993) Foraging strategy of wandering albatrosses through the breeding season: a study using satellite telemetry. Auk 110:325-342

Weimerskirch H, Brothers N, Jouventin P (1997a) Population dynamics of wandering albatross Diomedea exulans and Amsterdam albatross $D$. amsterdamensis in the Indian Ocean and their relationships with long-line fisheries: conservation implications. Biol Conserv 79:257-270

Weimerskirch H, Cherel Y, Cuénot-Chaillet F, Ridoux V (1997b) Alternative foraging strategies and resource allocation by male and female wandering albatrosses. Ecology 78:2051-2063

Weimerskirch H, Capdeville D, Duhamel G (2000) Factors

Editorial responsibility: Rory Wilson,

Swansea, UK affecting the number and mortality of seabirds attending trawlers and long-liners in the Kerguelen area. Polar Biol 23:236-249

*Weimerskirch H, Cherel Y, Delord K, Jaeger A, Patrick SC, Riotte-lambert L (2014) Lifetime foraging patterns of the wandering albatross: life on the move! J Exp Mar Biol Ecol 450:68-78

Weimerskirch H, Delord K, Guitteaud A, Phillips RA, Pinet P (2015) Extreme variation in migration strategies between and within wandering albatross populations during their sabbatical year, and their fitness consequences. Sci Rep 5:8853

Wilcox C, Van Sebille E, Hardesty BD (2015) Threat of plastic pollution to seabirds is global, pervasive, and increasing. Proc Natl Acad Sci USA 112:11899-11904

Williams R, McEldowney A (1990) A guide to the fish otoliths from waters off the Australian Antarctic Territory, Heard and Macquarie Islands. ANARE Res Notes 75:1-173

Wilson RP (1984) An improved stomach pump for penguins and other seabirds. J Field Ornithol 55:109-112

* Wood AG, Naef-Daenzer B, Prince PA, Croxall JP (2000) Quantifying habitat use in satellite-tracked pelagic seabirds: application of kernel estimation to albatross locations. J Avian Biol 31:278-286

Xavier JC, Cherel Y (2009) Cephalopod beak guide for the Southern Ocean. British Antarctic Survey, Cambridge

Xavier JC, Croxall JP (2007) Predator-prey interactions: Why do larger albatrosses eat bigger squid? J Zool (Lond) 271:408-417

* Xavier JC, Croxall JP, Trathan PN, Rodhouse PG (2003a) Inter-annual variation in the cephalopod component of the diet of the wandering albatross, Diomedea exulans, breeding at Bird Island, South Georgia. Mar Biol 142: 611-622

Xavier JC, Croxall JP, Trathan PN, Wood AG (2003b) Feeding strategies and diets of breeding grey-headed and wandering albatrosses at South Georgia. Mar Biol 143: 221-232

Xavier JC, Trathan PN, Croxall JP, Wood AG, Podesta G, Rodhouse PG (2004) Foraging ecology and interactions with fisheries of wandering albatrosses (Diomedea exulans) breeding at South Georgia. Fish Oceanogr 13: 324-344

Kavier JC, Croxall JP, Cresswell KA (2005) Boluses: an effective method for assessing the proportions of cephalopods in the diet of albatrosses. Auk 122:1182-1190

*Xavier JC, Phillips RA, Cherel Y (2011) Cephalopods in marine predator diet assessments: why identifying upper and lower beaks is important. ICES J Mar Sci 68: 1857-1864

Kavier JC, Walker K, Elliot G, Cherel Y, Thompson D (2014) Cephalopod fauna of South Pacific waters: new information from breeding New Zealand wandering albatrosses. Mar Ecol Prog Ser 513:131-142

* Young LC, Vanderlip C, Duffy DC, Afanasyev V, Shaffer SA (2009) Bringing home the trash: Do colony-based differences in foraging distribution lead to increased plastic ingestion in Laysan albatrosses? PLOS ONE 4:e7623

Young RE, Vecchione M, Mangold KM (2016) Cephalopoda Cuvier 1797. Octopods, squids, nautiluses, etc. Version 27 February 2016. Available at http://tolweb.org/ Cephalopoda/19386/2016.02.27 in The Tree of Life Web Project, http://tolweb.org/

Submitted: July 25, 2016; Accepted: November 23, 2016

Proofs received from author(s): January 26, 2017 